\title{
A semantical framework for hybrid knowledge bases
}

\author{
Jos de Bruijn · David Pearce • Axel Polleres • \\ Agustín Valverde
}

\begin{abstract}
In the ongoing discussion about combining rules and ontologies on the Semantic Web a recurring issue is how to combine first-order classical logic with nonmonotonic rule languages. Whereas several modular approaches to define a combined semantics for such hybrid knowledge bases focus mainly on decidability issues, we tackle the matter from a more general point of view. In this paper, we show how Quantified Equilibrium Logic (QEL) can function as a unified framework which embraces classical logic as well as disjunctive logic programs under the (open) answer set semantics. In the proposed variant of QEL, we relax the unique names assumption, which was present in earlier versions of QEL. Moreover, we show that this framework elegantly captures the existing modular approaches for hybrid knowledge bases in a unified way.
\end{abstract}

Keywords Hybrid knowledge bases - Ontologies - Nonmonotonic rules · Semantic web . Logic programming · Quantified equilibrium logic - Answer set programming

\section{Introduction}

In the current discussions on the Semantic Web architecture a recurring issue is how to combine a first-order classical theory formalising an ontology with a (possibly nonmonotonic) rule base. Nonmonotonic rule languages have received considerable attention and achieved 
maturity over the last few years especially due to the success of Answer Set Programming (ASP), a nonmonotonic, purely declarative logic programming and knowledge representation paradigm with many useful features such as aggregates, weak constraints and priorities, supported by efficient implementations (for an overview see [1]).

As a logical foundation for the answer set semantics and a tool for logical analysis in ASP, the system of Equilibrium Logic was presented by Pearce [24] and further developed in subsequent works (see [25] for an overview and references). The aim of this paper is to show how Equilibrium Logic can be used as a logical foundation for the combination of ASP and ontologies.

In the quest to provide a formal underpinning for a nonmonotonic rules layer for the Semantic Web which can coexist in a semantically well-defined manner with the Ontology layer, various proposals for combining classical first-order logic with different variants of ASP have been presented in the literature. ${ }^{1}$ We distinguish three kinds of approaches: At one end of the spectrum there are approaches which provide an entailment-based query interface to the Ontology in the bodies of ASP rules, resulting in a loose integration (e.g., [10,9]). At the other end there are approaches which use a unifying nonmonotonic formalism to embed both the Ontology and the rule base (e.g., [4, 23]), resulting in a tight coupling. Hybrid approaches (e.g., $[29-31,16]$ ) fall between these extremes. Common to hybrid approaches is the definition of a modular semantics based on classical first-order models, on the one hand, and stable models-often, more generally, referred to as answer sets ${ }^{2}$-on the other hand. Additionally, they require several syntactical restrictions on the use of classical predicates within rules, typically driven by considerations upon retaining decidability of reasoning tasks such as knowledge base satisfiability and predicate subsumption. With further restrictions of the classical part to decidable Description Logics (DLs), these semantics support straightforward implementation using existing DL reasoners and ASP engines, in a modular fashion. In this paper, we focus on such hybrid approaches, but from a more general point of view.

Example 1 Consider a hybrid knowledge base consisting of a classical theory $\mathcal{T}$ :

$$
\begin{aligned}
& \forall x . P E R S O N(x) \rightarrow(A G E N T(x) \wedge(\exists y \cdot H A S-M O T H E R(x, y))) \\
& \forall x .(\exists y \cdot H A S-M O T H E R(x, y)) \rightarrow A N \operatorname{MAL}(x)
\end{aligned}
$$

which says that every $P E R S O N$ is an $A G E N T$ and has some (unknown) mother, and everyone who has a mother is an $A N I M A L$, and a nonmonotonic $\operatorname{logic}$ program $\mathcal{P}$ :

$$
\begin{aligned}
& P E R S O N(x) \leftarrow A G E N T(x), \neg \text { machine }(x) \\
& A G E N T(\text { Dave Bowman })
\end{aligned}
$$

which says that $A G E N T$ s are by default PERSONs, unless known to be machines, and Dave Bowman is an AGENT.

Using such a hybrid knowledge base consisting of $\mathcal{T}$ and $\mathcal{P}$, we intuitively would conclude that PERSON (Dave Bowman) holds since he is not known to be a machine, and furthermore we would conclude that Dave Bowman has some (unknown) mother, and thus ANIMAL(Dave Bowman).

\footnotetext{
${ }^{1}$ Most of these approaches focus on the Description Logics fragments of first-order logic underlying the Web Ontology Language OWL.

2 "answer sets" denote the extension of stable models, which originally have only been defined for normal logic programs to more general logic progtams such as disjunctive programs.
} 
We see two important shortcomings in current hybrid approaches:

(1) Current approaches to hybrid knowledge bases differ not only in terms of syntactic restrictions, motivated by decidability considerations, but also in the way they deal with more fundamental issues which arise when classical logic meets ASP, such as the domain closure and unique names assumptions. ${ }^{3}$ In particular, current proposals implicitly deal with these issues by either restricting the allowed models of the classical theory or by using variants of the traditional answer set semantics which cater for open domains and nonunique names. So far, little effort has been spent in a comparing the approaches from a more general perspective. In this paper we aim to provide a generic semantic framework for hybrid knowledge bases that neither restricts models (e.g., to unique names) nor imposes syntactical restrictions driven by decidability concerns. (2) The semantics of current hybrid knowledge bases is defined in a modular fashion. This has the important advantage that algorithms for reasoning with this combination can be based on existing algorithms for DL and ASP satisfiability. A single underlying logic for hybrid knowledge bases which, for example, allows to capture notions of equivalence between combined knowledge bases in a standard way, is lacking though.

Our main contribution with this paper is twofold. First, we survey and compare different (extensions of the) answer set semantics, as well as the existing approaches to hybrid knowledge bases, all of which define nonmonotonic models in a modular fashion. Second, we propose to use Quantified Equilibrium Logic (QEL) as a unified logical foundation for hybrid knowledge bases: As it turns out, the equilibrium models of the combined knowledge base coincide exactly with the modular nonmonotonic models for all approaches we are aware of Refs. [16,29-31].

The remainder of this paper is structured as follows: Sect. 2 recalls some basics of classical first-order logic. Section 3 reformulates different variants of the answer set semantics introduced in the literature using a common notation and points out correspondences and discrepancies between these variants. Next, definitions of hybrid knowledge bases from the literature are compared and generalised in Sect. 4. QEL and its relation to the different variants of ASP are clarified in Sect. 5. Section 6 describes an embedding of hybrid knowledge bases into QEL and establishes the correspondence between equilibrium models and nonmonotonic models of hybrid $\mathrm{KBs}$. We discuss some immediate implications of our results in Sect. 7. In Sect. 8, we show how for finite knowledge bases an equivalent semantical characterisation can be given via a second-order operator NM. This behaves analogously to the operator SM used by Ferraris, Lee and Lifschitz [12] to define the stable models of a first-order sentence, except that its minimisation condition applies only to the nonclassical predicates. In Sect. 9 we discuss an application of the previous results: we propose a definition of strong equivalence for knowledge bases sharing a common structural language and show how this notion can be captured by deduction in the (monotonic) logic of here-andthere. These two Sects. 8 and 9 particularly contain mostly new material which has not yet been presented in the conference version [5] of this article. We conclude with a discussion of further related approaches and an outlook to future work in Sect. 10.

\section{First-order logic (FOL)}

A function-free first-order language $\mathcal{L}=\langle C, P\rangle$ with equality consists of disjoint sets of constant and predicate symbols $C$ and $P$. Moreover, we assume a fixed countably infinite set of variables, the symbols ' $\rightarrow$ ', ' $\vee$ ', ' $\wedge$ ', ' $\neg$ ', ' $\exists$ ', ' $\mathbf{y}^{\prime}$, and auxiliary parentheses ' (', ' ')', Each

$\overline{3}$ See [3] for a more in-depth discussion of these issues. 
predicate symbol $p \in P$ has an assigned arity $\operatorname{ar}(p)$. Atoms and formulas are constructed as usual. Closed formulas, or sentences, are those where each variable is bound by some quantifier. A theory $T$ is a set of sentences. Variable-free atoms, formulas, or theories are also called ground. If $D$ is a nonempty set, we denote by $A t_{D}(C, P)$ the set of ground atoms constructible from $\mathcal{L}^{\prime}=\langle C \cup D, P\rangle$.

Given a first-order language $\mathcal{L}$, an $\mathcal{L}$-structure consists of a pair $\mathcal{I}=\langle U, I\rangle$, where the universe $U=(D, \sigma)$ (sometimes called pre-interpretation) consists of a nonempty domain $D$ and a function $\sigma: C \cup D \rightarrow D$ which assigns a domain value to each constant such that $\sigma(d)=d$ for every $d \in D$. For tuples, we write $\sigma(\vec{t})=\left(\sigma\left(d_{1}\right), \ldots, \sigma\left(d_{n}\right)\right)$. We call $d \in D$ an unnamed individual if there is no $c \in C$ such that $\sigma(c)=d$. The function $I$ assigns a relation $p^{I} \subseteq D^{\prime \prime}$ to each $n$-ary predicate symbol $p \in P$ and is called the $\mathcal{L}$-interpretation over $D$. The designated binary predicate symbol $e q$, occasionally written ${ }^{\prime}={ }^{\prime}$ in infix notation, is assumed to be associated with the fixed interpretation function $e q^{I}=\{(d, d): d \in D\}$. If $\mathcal{I}$ is an $\mathcal{L}^{\prime}$-structure we denote by $\left.\mathcal{I}\right|_{\mathcal{L}}$ the restriction of $\mathcal{I}$ to a sublanguage $\mathcal{L} \subseteq \mathcal{L}^{\prime}$.

An $\mathcal{L}$-structure $\mathcal{I}=\langle U, I\rangle$ satisfies an atom $p\left(d_{1}, \ldots, d_{n}\right)$ of $A t_{D}(C, P)$, witten $\mathcal{I} \models p\left(d_{1}, \ldots, d_{n}\right)$, if $\left(\sigma\left(d_{1}\right), \ldots, \sigma\left(d_{n}\right)\right) \in p^{I}$. This is extended as usual to sentences and theories.

$\mathcal{I}$ is a model of an atom (sentence, theory, respectively) $\varphi$, written $\mathcal{I} \models \varphi$, if it satisfies $\varphi$. A theory $T$ entails a sentence $\varphi$, written $T \models \varphi$, if every model of $\mathcal{T}$ is also a model of $\varphi$. A theory is consistent if it has a model.

In the context of logic programs, the following assumptions often play a role: We say that the paraneter names assumption ( $P N A$ ) applies in case $\left.\sigma\right|_{C}$ is surjective, i.e, there are no unnamed individuals in $D$; the unique names assumption (UNA) applies in case $\left.\sigma\right|_{C}$ is injective; in case both the PNA and UNA apply, the standard names assumption (SNA) applies, i.e., $\left.\sigma\right|_{C}$ is a bijection. In the following, we will speak about PNA-, UNA-, or SNA-structures, (or PNA-, UNA-, or SNA-models, respectively), depending on $\sigma$.

An $\mathcal{L}$-interpretation $I$ over $D$ can be seen as a subset of $A t_{D}(C, P)$. So, we can define a subset relation for $\mathcal{L}$-structures $\mathcal{I}_{1}=\left\langle\left(D, \sigma_{1}\right), I_{1}\right\rangle$ and $\mathcal{I}_{2}=\left\langle\left(D, \sigma_{2}\right), I_{2}\right\rangle$ over the same domain by setting $\mathcal{I}_{1} \subseteq \mathcal{I}_{2}$ if $I_{1} \subseteq I_{2}{ }^{4}$ Whenever we speak about subset minimality of models/structures in the following, we thus mean minimality among all models/structures over the same domain.

\section{Answer set semantics}

In this paper, we assume nonground disjunctive logic programs with negation allowed in rule heads and bodies, interpreted under the answer set semantics [21]. ${ }^{5}$ A program $\mathcal{P}$ consists of a set of rules of the form

$$
a_{1} \vee a_{2} \vee \ldots \vee a_{k} \vee \neg a_{k+1} \vee \ldots \vee \neg a_{l} \leftarrow b_{1}, \ldots, b_{m}, \neg b_{m+1}, \ldots, \neg b_{n}
$$

where $a_{i}(i \in\{1, \ldots, l\})$ and $b_{j}(j \in\{1, \ldots, n\})$ are atoms, called head (body, respectively) atoms of the rule, in a function-free first-order language $\mathcal{L}=\langle C, P\rangle$ without equality. By $C_{\mathcal{P}} \subseteq C$, we denote the set of constants which appear in $\mathcal{P}$. A rule with $k=I$ and $m=n$ is called positive. Rules where each variable appears in $b_{1}, \ldots, b_{m}$ are called safe. A program is positive (safe) if all its rules are positive (safe).

\footnotetext{
4 Note that this is not the substructure or submodel relation in classical model theory, which holds between a structure and its restriction to a subdomain.

${ }^{5} \mathrm{By} \neg$ we mean negation as failure and not classical, or strong negation, which is also sometimes considered in ASP.
} 
For the purposes of this paper, we give a slightly generalised definition of the common notion of the grounding of a program: The grounding $\operatorname{sr}_{U}(\mathcal{P})$ of $\mathcal{P}$ wrt. a universe $U=(D, \sigma)$ denotes the set of all rules obtained as follows: For $r \in \mathcal{P}$, replace (i) each constant $c$ appearing in $r$ with $\sigma(c)$ and (ii) each variable with some element in $D$. Observe that thus $g r_{U}(\mathcal{P})$ is a ground program over the atoms in $A t_{D}(C, P)$.

For a ground program $\mathcal{P}$ and first-order structure $\mathcal{I}$, the reduct $\mathcal{P}^{\mathcal{I}}$ consists of rules

$$
a_{1} \vee a_{2} \vee \ldots \vee a_{k} \leftarrow b_{1}, \ldots, b_{m}
$$

obtained from all rules of the form (1) in $\mathcal{P}$ for which it holds that $\mathcal{I} \models a_{i}$ for all $k<i \leq l$ and $\mathcal{I} \not \models b_{j}$ for all $m<j \leq n$.

Answer set semantics is usually defined in terms of Herbrand structures over $\mathcal{L}=\langle C, P\}$. Herbrand structures have a fixed universe, the Herbrand universe $\mathcal{H}=(C, i d)$, where $i d$ is the identity function. For a Herbrand structure $\mathcal{I}=\langle\mathcal{H}, I\rangle, I$ can be viewed as a subset of the Hetbrand base, $\mathcal{B}$, which consists of the ground atoms of $\mathcal{L}$. Note that by detinition of $\mathcal{H}$, Herbrand structures are SNA-structures. A Herbrand structure $\mathcal{I}$ is an answer set [21] of $\mathcal{P}$ if $\mathcal{I}$ is subset minimal among the structures satisfying $g r_{\mathcal{H}}(\mathcal{P})^{\mathcal{I}}$. Two variations of this semantics, the open [15] and generalised open answer set [16] semantics, consider open domains, thereby relaxing the PNA. An extended Herbrand structure is a first-order structure based on a universe $U=(D, i d)$, where $D \supseteq C$.

Definition 1 A first-order $\mathcal{L}$-structure $\mathcal{I}=\langle U, I\rangle$ is called a generalised open answer set of $\mathcal{P}$ if $\mathcal{I}$ is subset minimal among the structures satisfying all rules in $g r_{U}(\mathcal{P})^{\mathcal{I}}$. If, additionally, $\mathcal{I}$ is an extended Herbrand structure, then $\mathcal{I}$ is an open answer set of $\mathcal{P}$.

In the open answer set semantics the UNA applies. We have the following correspondence with the answer set semantics. First, as a straightforward consequence from the definitions, we can observe:

Proposition 1 If $\mathcal{M}$ is an answer set of $\mathcal{P}$ then $\mathcal{M}$ is also an open answer set of $\mathcal{P}$.

The converse does not hold in general:

Example 2 Consider $\mathcal{P}=\{p(a) ; o k \leftarrow \neg p(x) ; \leftarrow \neg o k\}$ over $\mathcal{L}=\{\{a\},\{p, o k\}\}$. We leave it as an exercise to the reader to show that $\mathcal{P}$ is inconsistent under the answer set semantics, but $\mathcal{M}=\left\{\left(\left\{a, c_{1}\right\}, i d\right),\{p(a), o k\}\right\}$ is an open answer set of $\mathcal{P}$.

Open answer set programs allow the use of the equality predicate '=' in the body of rules. However, since this definition of open answer sets adheres to the UNA, one could argue that equality in open answer set programming is purely syntactical. Positive equality predicates in rule bodies can thus be eliminated by simple pre-processing, applying unification. This is not the case for negative occurrences of equality, but, since the interpretation of equality is fixed, these can be eliminated during grounding.

An alternative approach to relax the UNA has been presented by Rosati in Ref. [30]: Instead of grounding with respect to $U$, programs are grounded with respect to the Herbrand universe $\mathcal{H}=(C, i d)$, and minimality of the models of $g \boldsymbol{H}_{\mathcal{H}}(\mathcal{P})^{\mathcal{I}}$ wrt. $U$ is redefined: $\mathcal{I}_{\left.\right|_{\mathcal{H}}}=\left\{p\left(\sigma\left(c_{1}\right), \ldots, \sigma\left(c_{n}\right)\right): p\left(c_{1}, \ldots, c_{H}\right) \in \mathcal{B}, \mathcal{I} \equiv p\left(c_{1}, \ldots, c_{n}\right)\right\}$, i.e., $\mathcal{I}_{\left.\right|_{\mathcal{H}}}$ is the restriction of $\mathcal{I}$ to ground atoms of $\mathcal{B}$. Given $\mathcal{L}$-structures $\mathcal{I}_{1}=\left(U_{1}, I_{1}\right)$ and $\mathcal{I}_{2}=\left(U_{2}, I_{2}\right){ }^{6}$ the relation $\mathcal{I}_{1} \subseteq \mathcal{H} \mathcal{I}_{2}$ holds if $\left.\left.\mathcal{I}_{1}\right|_{\mathcal{H}} \subseteq \mathcal{I}_{2}\right|_{\mathcal{H}}$.

${ }^{6}$ Not necessarily over the same domain. 
Definition 2 An $\mathcal{L}$-structure $\mathcal{I}$ is called a generalised answer set of $\mathcal{P}$ if $\mathcal{I}$ is $\subseteq \mathcal{H}^{- \text {minimal }}$ among the structures satisfying all rules in $g r_{\mathcal{H}}(\mathcal{P})^{\mathcal{I}}$.

The following Lemma (implicit in [14]) establishes that, for safe programs, all atoms of $A t_{D}(C, P)$ satisfied in an open answer set of a safe program are ground atoms over $C_{P}$ :

Lemma 2 Let $\mathcal{P}$ be a safe program over $\mathcal{L}=\langle C, P\rangle$ with $\mathcal{M}=\langle U, I\rangle$ a (generalised) open answer set over universe $U=(D, \sigma)$. Then, for any atom from $A t_{D}(C, P)$ such that $\mathcal{M} \vDash p\left(d_{1}, \ldots, d_{n}\right)$, there exist $c_{i} \in C_{p}$ such that $\sigma\left(c_{i}\right)=d_{i}$ for each $1 \leq i \leq n$.

Proof First, we observe that any atom $\mathcal{M} \models p\left(d_{1}, \ldots, d_{n}\right)$ must be derivable from a sequence of rules $\left(r_{0} ; \ldots ; r_{l}\right)$ in $g r_{U}(\mathcal{P})^{\mathcal{M}}$. We prove the lemma by induction over the length $I$ of this sequence. $I=0$ : Assume $\mathcal{M} \models p\left(d_{1}, \ldots, d_{n}\right)$, then $r_{0}$ must be (by safety) a ground fact in $\mathcal{P}$ such that $p\left(\sigma\left(c_{1}\right), \ldots, \sigma\left(c_{n}\right)\right)=p\left(d_{1}, \ldots, d_{n}\right)$ and $c_{1}, \ldots, c_{n} \in C_{\mathcal{P}}$. As for the induction step, let $p\left(d_{1}, \ldots, d_{n}\right)$ be inferred by application of rule $n_{l} \in g r_{U}(\mathcal{P})^{\mathcal{M}}$. By safety, again each $d_{j}$ either stems from a constant $c_{j} \in C_{P}$ such that $\sigma\left(c_{j}\right)=d_{j}$ which appears in some true head atom of $n_{l}$ or $d_{j}$ also appears in a positive body atom $q\left(\ldots, d_{j}, \ldots\right)$ of $r_{l}$ such that $\mathcal{M} \models q\left(\ldots, d_{j}, \ldots\right)$, derivable by $\left(r_{0} ; \ldots ; r_{l-1}\right)$, which, by the induction hypothesis, proves the existence of a $c_{j} \in C_{p}$ with $\sigma\left(c_{j}\right)=d_{j}$.

From this Lemma, the following correspondence follows directly. Note that the answer sets and open answer sets of safe programs coincide as a direct consequence of Lemma 2 :

Proposition $3 \mathcal{M}$ is an answer set of a safe program $\mathcal{P}$ if and only if $\mathcal{M}$ is an open answer set of $\mathcal{P}$.

Similarly, on unsafe programs, generalised answer sets and generalised open answer sets do not necessarily coincide, as demonstrated by example 2 . However, the following correspondence follows straightforwardly from Lemma 2 :

Proposition 4 Given a safe program $\mathcal{P}, \mathcal{M}$ is a generalised open answer set of $\mathcal{P}$ if and only if $M$ is a generalised answer set of $P$.

\section{Proof}

( $\Rightarrow$ ) Assume $\mathcal{M}$ is a generalised open answer set of $\mathcal{P}$. By Lemma 2, we know that rules in $g r_{U}(\mathcal{P})^{\mathcal{M}}$ involving unnamed individuals do not contribute to answer sets, since their body is always false. It follows that $\mathcal{M}=\left.\mathcal{M}\right|_{\mathcal{H}}$ which in turn is a $\subseteq_{\mathcal{H}}$-minimal model of $g r_{\mathcal{H}}(\mathcal{P})^{\mathcal{M}}$. This follows from the observation that each rule in $g r_{\mathcal{H}}(\mathcal{P})^{\mathcal{M}}$ and its corresponding rules in $g r_{U}(\mathcal{P})^{\mathcal{M}}$ are satisfied under the same models.

$(\Leftarrow)$ Analogously.

By similar arguments, generalised answer sets and generalised open answer sets coincide in case the parameter name assumption applies:

Proposition 5 Let $\mathcal{M}$ be a PNA-structure. Then $\mathcal{M}$ is a generalised answer set of $\mathcal{P}$ if and only if $\mathcal{M}$ is a generalised open answer of $\mathcal{P}$.

If the SNA applies, consistency with respect to all semantics introduced so far boils down to consistency under the original definition of answer sets:

Proposition 6 A program $\mathcal{P}$ has an answer set if and only if $\mathcal{P}$ has a generalised open answer set under the SNA. 
Answer sets under SNA may differ from the original answer sets since also non-Herbrand structures are allowed. Further, we observe that there are programs which have generalised (open) answer sets but do not have (open) answer sets, even for safe programs, as shown by the following simple example:

Example 3 Consider $\mathcal{P}=\{p(a) ; \leftarrow \neg p(b)\}$ over $\mathcal{L}=\{\{a, b\},\{p\}\}$. $\mathcal{P}$ is ground, thus obviously safe. However, although $\mathcal{P}$ has a generalised (open) answer set-the reader may verify this by, for instance, considering the one-element universe $U=(\{d\}, \sigma)$, where $\sigma(a)=\sigma(b)=d$-it is inconsistent under the open answer set semantics, i.e., the program does not have any open (nongenrealised) answer set.

\section{Hybrid knowledge bases}

We now turn to the concept of hybrid knowledge bases, which combine classical theories with the various notions of answer sets. We define a notion of hybrid knowledge bases which generalises definitions in the literature $[16,29-31]$. We then compare and discuss the differences between the various definitions. It turns out that the differences are mainly concerned with the notion of answer sets, and syntactical restrictions, but do not change the general semantics. This will allow us to base our embedding into Quantified Equilibrium Logic on a unified definition.

A hybrid knowledge base $\mathcal{K}=(\mathcal{T}, \mathcal{P})$ over the function-free language $\mathcal{L}=\left\langle C, P_{\mathcal{T}} \cup P_{\mathcal{P}}\right\rangle$ consists of a classical first-order theory $T$ (also called the structural part of $\mathcal{K}$ ) over the language $\mathcal{L}_{T}=\left\langle C, P_{T}\right\rangle$ and a program $\mathcal{P}$ (also called rules part of $\mathcal{K}$ ) over the language $\mathcal{L}$, where $P_{T} \cap P_{P}=\emptyset$, i.e., $\mathcal{T}$ and $\mathcal{P}$ share a single set of constants, and the predicate symbols allowed to be used in $\mathcal{P}$ are a superset of the predicate symbols in $\mathcal{L}_{\mathcal{T}}$. Intuitively, the predicates in $\mathcal{L}_{T}$ are interpreted classically, whereas the predicates in $\mathcal{L}_{\mathcal{P}}$ are interpreted nonmonotonically under the (generalised open) answer set semantics. With $C_{P}=\left\langle C, P_{P}\right\rangle$ we denote the restricted language of $\mathcal{P}$ to only the distinct predicates $P_{P}$ which are not supposed to occur in $\mathcal{T}$.

We do not consider the alternative classical semantics defined in Refs. [29-31], as these are straightforward.

We define the projection of a ground program $\mathcal{P}$ with respect to an $\mathcal{L}$-structure $\mathcal{I}=\langle U, I\rangle$, denoted $\Pi(\mathcal{P}, \mathcal{I})$, as follows: for each rule $r \in \mathcal{P}, r^{\Pi}$ is defined as:

1. $r^{\Pi}=\emptyset$ if there is a literal over $A t_{D}\left(C, P_{T}\right)$ in the head of $r$ of form $p(\bar{t})$ such that $p(\sigma(\bar{t})) \in I$ or of form $\neg p(\bar{t})$ with $p(\sigma(\bar{t})) \notin I$;

2. $r^{\Pi}=\emptyset$ if there is a literal over $A t_{D}\left(C, P_{T}\right)$ in the body of $r$ of form $p(\bar{t})$ such that $p(\sigma(\vec{t})) \notin I$ or of form $\neg p(\bar{t})$ such that $p(\sigma(\bar{t})) \in I$;

3. otherwise $r^{\Pi}$ is the singleton set resulting from $r$ by deleting all occurrences of literals from $\mathcal{L}_{T}$,

and $\Pi(\mathcal{P}, \mathcal{I})=\bigcup\left\{r^{\Pi}: r \in \mathcal{P}\right\}$. Intuitively, the projection "evaluates" all classical literals in $\mathcal{P}$ with respect to $\mathcal{I}$.

Definition 3 Let $\mathcal{K}=(T, \mathcal{P})$ be a hybrid knowledge base over the language $\mathcal{L}=\left\langle C, P_{T} \mathrm{U}\right.$ $\left.P_{\mathcal{P}}\right\}$. An NM-model $\mathcal{M}=\langle U, I\rangle$ (with $U=(D, \sigma)$ ) of $\mathcal{K}$ is a first-order $\mathcal{L}$-structure such that $\left.\mathcal{M}\right|_{\mathcal{L}_{T}}$ is a model of $\mathcal{T}$ and $\left.\mathcal{M}\right|_{\mathcal{L}_{\mathcal{F}}}$ is a generalised open answer set of $\Pi\left(\mathrm{gr}_{U}(\mathcal{P}), \mathcal{M}\right)$.

Analogous to first-order models, we speak about PNA-, UNA-, and SNA-NM-models. 
Example 4 Consider the hybrid knowledge base $\mathcal{K}=(T, \mathcal{P})$, with $T$ and $\mathcal{P}$ as in Example 1, with the capitalised predicates being predicates in $P_{T}$. Now consider the interpretation $\mathcal{I}=\{U, I\}$ (with $U=(D, \sigma)$ ) with $D=\{$ Dave Bowman, $k\}, \sigma$ the identity function, and $I=\{A G E N T$ (DaveBowman), HAS-MOTHER(DaveBowman, $k$ ),

ANIMAL(Dave Bowman), machine (DaveBowman)\}. Clearly, $\left.\mathcal{I}\right|_{\mathcal{L}_{T}}$ is a model of $\mathcal{T}$. The projection $\Pi\left(g_{U}(\mathcal{P}), \mathcal{I}\right)$ is

\section{$\leftarrow \neg$ machine (Dave Bowman),}

which does not have a stable model, and thus $\mathcal{I}$ is not an NM-model of $\mathcal{K}$. In fact, the logic program $\mathcal{P}$ ensures that an interpretation cannot be an NM-model of $\mathcal{K}$ if there is an $A G E N T$ which is neither a $P E R S O N$ nor known (by conclusions from $\mathcal{P}$ ) to be a machine. It is easy to verify that, for any NM-model of $\mathcal{K}$, the atoms $A G E N T$ (Dave Bowman), PERSON (DaveBowman), and ANIMAL(DaveBowman) must be true, and are thus entailed by $\mathcal{K}$. The latter cannot be derived from neither $\mathcal{T}$ nor $\mathcal{P}$ individually.

\section{1 r-hybrid KBs}

We now proceed to compare our definition of NM-models with the various definitions in the literature. The first kind of hybrid knowledge base we consider was introduced by Rosati in Ref. [29] (and extended in Ref. [31] under the name $\mathcal{D} \mathcal{L}+\log$ ), and was labelled r-hybrid knowledge base. Syntactically, r-hybrid $\mathrm{KBs}$ do not allow negated atoms in rule heads, i.e., for rules of the form(1) $l=k$, and do not allow atoms from $\mathcal{L}_{\mathcal{T}}$ to occur negatively in the rule body. ${ }^{7}$ Moreover, in Ref. [29], Rosati deploys a restriction which is stronger than standard safety: each variable must appear in at least one positive body atom with a predicate from $\mathcal{L}_{\mathcal{P}}$. We call this condition $\mathcal{L}_{\mathcal{P}}$-safe in the remainder. In Ref. [31] this condition is relaxed to weak $\mathcal{L}_{\mathcal{P}}$-safety: there is no special safety restriction for variables which occur only in body atoms from $P_{T}$.

Semantically, Rosati assumes (an infinite number of) standard names, i.e., $C$ is countably infinite, and normal answer sets, in his version of NM-models:

Definition 4 Let $\mathcal{K}=(T, \mathcal{P})$ be an r-hybrid knowledge base, over the language $\mathcal{L}=\left\langle C, P_{\mathcal{T}} \cup P_{\mathcal{P}}\right\rangle$, where $\mathcal{C}$ is countably infinite, and $\mathcal{P}$ is a (weak) $\mathcal{L}_{P}$-safe program. An $r-N M$-model $\mathcal{M}=\langle U, I\rangle$ of $\mathcal{K}$ is a first-order $\mathcal{C}$-SNA-structure such that $\left.\mathcal{M}\right|_{\mathcal{C}_{T}}$ is a model of $\mathcal{T}$ and $\left.\mathcal{M}\right|_{\mathcal{L}_{\mathcal{P}}}$ is an answer set of $\Pi(g r v(\mathcal{P}), \mathcal{M})$.

In view of the (weak) $\mathcal{L}_{p}$-safety condition, we observe that r-NM-model existence coincides with SNA-NM-model existence on r-hybrid knowledge bases, by Lemma 2 and Proposition 6.

The syntactic restrictions in r-hybrid knowledge bases guarantee decidability of the satisfiability problem in case satisfiability (in case of $\mathcal{L}_{P}$-safety) or conjunctive query containment (in case of weak $\mathcal{L} p$-safety) in $\mathcal{T}$ is decidable. Rosati $[29,31]$ presents sound and complete algorithms for both cases.

7 Note that by projection, negation of predicates from $P_{T}$ is treated classically, whereas negation of predicates from $P_{p}$ is treated nonmonotonically. This might be considered unintuitive and therefore a reason why Rosati disallows structural predicates to occut negated. The negative occurtence of classical predicates in the body is eguivalent to the positive occurtence of the predicate in the head. 
Table 1 Different vatiants of hybrid KBs

\begin{tabular}{lllll}
\hline & SNA & Variables & Disjunctive rule beads & Negated LT atoms \\
\hline r-hybrid & Yes & $\mathcal{L}$ p-safe & Pos. only & No \\
$\mathbf{r}^{+}$-hybrid & No & $\mathcal{L}$ - -safe & Pos. only & No \\
$\mathbf{r}_{w}$-hybrid & Yes & Weak $\mathcal{L}_{P \text {-safe }}$ & Pos. only & No \\
g-hybrid & No & Guarded & Neg. allowed & Yes \\
\hline
\end{tabular}

* g-hybrid allows negation in the head but at most one positive head atom

\section{$4.2 \mathrm{r}^{+}$-hybrid KBs}

In Ref. [30], Rosati relaxes the UNA for what we will call here $\mathrm{r}^{+}$-hybrid knowledge bases. In this variant the $\mathcal{L}_{P}$-safety restriction is kept but generalised answer sets under arbitrary interpretations are considered:

Definition 5 Let $\mathcal{K}=(T, \mathcal{P})$ be an $\mathrm{r}^{+}$-hybrid knowledge base consisting of a theory $\mathcal{T}$ and an $\mathcal{L}$ - -safe program $\mathcal{P}$. An $\mathrm{r}^{+}-N M$-model, $\mathcal{M}=\langle U, I\rangle$ of $\mathcal{K}$ is a first-order $\mathcal{L}$-structure such that $\left.\mathcal{M}\right|_{\mathcal{L}_{T}}$ is a model of $T$ and $\left.\mathcal{M}\right|_{\mathcal{C}_{p}}$ is a generalised answer set of $\Pi\left(\mathrm{gr}_{U}(\mathcal{P}), \mathcal{M}\right)$.

$\mathcal{L}_{\mathcal{P}}$-safety guarantees safety of $\Pi\left(g r_{U}(\mathcal{P}), \mathcal{M}\right)$. Thus, by Proposition 3 , we can conclude that $r^{+}$-NM-models coincide with NM-models on $r$-hybrid knowledge bases. The relaxation of the UNA does not affect decidability.

\section{3 g-hybrid KBs}

G-hybrid knowledge bases [16] allow a different form of rules in the program. In order to regain decidability, rules are not required to be safe, but they are required to be guarded (hence the ' $g$ ' in $g$-hybrid): All variables in a rule are required to occur in a single positive body atom, the guard, with the exception that unsafe choice rules of the form

$$
p\left(c_{1}, \ldots, c_{n}\right) \vee \neg p\left(c_{1}, \ldots, c_{n}\right) \leftarrow
$$

are allowed. Moreover, disjunction in rule heads is limited to at most one positive atom, i.e., for rules of the form( 1 ) we have that $k \leq 1$, but an arbitrary number of negated head atoms is allowed. Another significant difference is that, as opposed to the approaches based on r-hybrid KBs, negative structural predicates are allowed in the rules part within g-hybrid knowledge bases (see also Footnote 7). The definition of NM-models in Ref. [16] coincides precisely with our Definition 3.

Table 1 summarises the different versions of hybrid knowledge bases introduced in the literature.

\section{Quantified equilibrium logic (QEL)}

Equilibrium logic for propositional theories and logic programs was presented in Ref. [24] as a foundation for answer set semantics, and extended to the first-order case in Ref. [26], as well as, in slightly more general, modified form, in Ref. [27]. For a survey of the main properties of equilibrium logic, see [25]. Usually in quantified equilibrium logic, we consider a full firstorder language allowing function symbols and we include a second, strong negation operator 
as occurs in several ASP dialects. For the present purpose of drawing comparisons with approaches to hybrid knowledge bases, it will suffice to consider the function-free language with a single negation symbol, ' $\neg$ '. In particular, we shall work with a quantified version of the logic HT of here-and-there. In other respects, we follow the treatment of Ref. [27].

\subsection{General structures for quantified here-and-there logic}

As before, we consider a function-free first order language $\mathcal{L}=\{C, P\rangle$ built over a set of constant symbols, $C$, and a set of predicate symbols, $P$. The sets of $\mathcal{L}$-formulas, $\mathcal{L}$-sentences and atomic $\mathcal{L}$-sentences are defined in the usual way. Again, we only work with sentences, and, as in Sect. 2, by an $\mathcal{L}$-interpretation $I$ over a set $D$ we mean a subset $I$ of At $D(C, P)$. A here-and-there $\mathcal{L}$-structure with static domains, or $\mathbf{Q H T}^{\mathcal{S}}(\mathcal{L})$-structure, is a tuple $\mathcal{M}=\left\langle(D, \sigma), I_{h}, I_{l}\right\}$ where $\left\{(D, \sigma), I_{h}\right\}$ and $\left\{(D, \sigma), I_{t}\right\}$ are $\mathcal{L}$-structures such that $I_{h} \subseteq I_{t}$.

We can think of $\mathcal{M}$ as a structure similar to a first-order classical model, but having two parts, or components, $h$ and $t$ that correspond to two different points or "worlds", 'here' and 'there', in the sense of Kripke semantics for intuitionistic logic [32], where the worlds are ordered by $h \leq t$. At each world $w \in\{h, t\}$, one verifies a set of atoms $I_{w}$ in the expanded language for the domain $D$. We call the model static, since, in contrast to say intuitionistic logic, the same domain serves each of the worlds. ${ }^{8}$ Since $h \leq t$, whatever is verified at $h$ remains true at $t$. The satisfaction relation for $\mathcal{M}$ is defined so as to reflect the two different components, so we write $\mathcal{M}, w \models \varphi$ to denote that $\psi$ is true in $\mathcal{M}$ with respect to the $w$ component. Evidently we should require that an atomic sentence is true at $w$ just in case it belongs to the $w$-interpretation. Formally, if $p\left(t_{1}, \ldots, t_{n}\right) \in \mathrm{At}_{D}$ then

$$
\mathcal{M}, w \models p\left(t_{1}, \ldots, t_{n}\right) \text { iff } p\left(\sigma\left(t_{1}\right), \ldots, \sigma\left(t_{n}\right)\right) \in I_{w} .
$$

Then, $\models$ is extended recursively as follows ${ }^{9}$ :

$-\mathcal{M}, w \models \varphi \wedge \psi$ iff $\mathcal{M}, w \models \varphi$ and $\mathcal{M}, w \models \psi$.

- $\mathcal{M}, w \models \varphi \vee \psi$ iff $\mathcal{M}, w \models \varphi$ or $\mathcal{M}, w \models \psi$.

- $\mathcal{M}, t \models \varphi \rightarrow \psi$ iff $\mathcal{M}, t \models \varphi$ or $\mathcal{M}, t \models \psi$.

- $\mathcal{M}, h \models \varphi \rightarrow \psi$ iff $\mathcal{M}, t \models \varphi \rightarrow \psi$ and $\mathcal{M}, h \models \varphi$ or $\mathcal{M}, h \models \psi$.

- $\mathcal{M}, w \models \neg \varphi$ iff $\mathcal{M}, t \notin \varphi$.

- $\mathcal{M}, t \models \forall x \psi(x)$ iff $\mathcal{M}, t \models \varphi(d)$ for all $d \in D$.

- $\mathcal{M}, h \models \forall x \varphi(x)$ iff $\mathcal{M}, t \models \forall x \varphi(x)$ and $\mathcal{M}, h \models \varphi(d)$ for all $d \in D$.

- $\mathcal{M}, w \models \exists x \varphi(x)$ iff $\mathcal{M}, w \models \varphi(d)$ for some $d \in D$.

Truth of a sentence in a model is defined as follows: $\mathcal{M} \models \varphi$ iff $\mathcal{M}, w \models \varphi$ for each $w \in\{h, t\}$. A sentence $\varphi$ is valid if it is true in all models, denoted by $\models \varphi$. A sentence $\psi$ is a consequence of a set of sentences $\Gamma$, denoted $\Gamma \vDash \psi$, if every model of $\Gamma$ is a model of $\psi$. In a model $\mathcal{M}$ we often use the symbols $H$ and $T$, possibly with subscripts, to denote the interpretations $I_{h}$ and $I_{t}$ respectively; so, an $\mathcal{L}$-structure may be written in the form $\{U, H, T\}$, where $U=(D, \sigma)$.

The resulting logic is called Quantified Here-and-There Logic with static domains, denoted by QHT $^{s}$. In terms of satisfiability and validity this logic is equivalent to the logic introduced

\footnotetext{
8 Alternatively it is quite common to speak of a logic with constant domains. However this is slightly ambignous since it might suggest that the domain is composed only of constants, which is not intended hete.

9 The reader may easily check that the following correspond exactly to the usual Kripke semantics for intuitionistic logic given out assumptions about the two worlds $h$ and $t$ and the single domain $D$, see e.g., [32].
} 
before in Ref. [26]. By $\mathbf{Q H T}_{=}^{s}$ we denote the version of QEL with equality. The equality predicate in $\mathbf{Q H T}_{=}^{s}$ is interpreted as the actual equality in both worlds, ie $\mathcal{M}, w \models t_{1}=t_{2}$ iff $\sigma\left(t_{1}\right)=\sigma\left(t_{2}\right)$.

The logic $\mathbf{Q H T}_{=}^{s}$ can be axiomatised as follows. Let $\mathbf{I N T}^{=}$denote first-order intuitionistic logic [32] with the usual axioms for equality:

$$
\begin{aligned}
& x=x, \\
& x=y \rightarrow(F(x) \rightarrow F(y)),
\end{aligned}
$$

for every formula $F(x)$ such that $y$ is substitutable for $x$ in $F(x)$. To this we add the axiom of Hosoi

$$
\alpha \vee(\neg \beta \vee(\alpha \rightarrow \beta)),
$$

which determines 2-element here-and-there models in the propositional case, and the axiom SQHT (static quantified here-and-there):

$$
\exists x(F(x) \rightarrow \forall x F(x))
$$

Lastly we add the "decidable equality" axiom:

$$
x=y \vee x \neq y .
$$

For a completeness proof for $\mathbf{Q H T}_{=}^{s}$, see [20].

As usual in first order logic, satisfiability and validity are independent from the language. If $\mathcal{M}=\langle(D, \sigma), H, T\rangle$ is an $\mathbf{Q H T}_{=}^{s}\left(\mathcal{L}^{\prime}\right)$-structure and $\mathcal{L} \subset \mathcal{L}^{\prime}$, we denote by $\left.\mathcal{M}\right|_{\mathcal{L}}$ the restriction of $\mathcal{M}$ to the sublanguage $\overline{\mathcal{L}}:\left.\mathcal{M}\right|_{\mathcal{L}}=\left\langle\left(D,\left.\sigma\right|_{\mathcal{L}}\right),\left.H\right|_{\mathcal{L}},\left.T\right|_{\mathcal{L}}\right\rangle$.

Proposition 7 Suppose that $\mathcal{L}^{\prime} \supset \mathcal{L}, \Gamma$ is a theory in $\mathcal{L}$ and $\mathcal{M}$ is an $\mathcal{L}^{\prime}$-structure such $\mathcal{M} \vDash \Gamma$. Then $\left.\mathcal{M}\right|_{\mathcal{L}}$ is a model of $\Gamma$ in $\mathbf{Q H T}_{=}^{s}(\mathcal{L})$.

Proposition 8 Suppose that $\mathcal{L}^{\prime} \supset \mathcal{L}$ and $\varphi \in \mathcal{L}$. Then $\varphi$ is valid (resp. satisfiable) in $\mathbf{Q H T}_{=}^{s}(\mathcal{L})$ if and only if is ralid (resp. satisfiable) in $\mathbf{Q H T}_{=}^{s}\left(\mathcal{L}^{\prime}\right)$.

Analogous to the case of classical models, we can define special kinds of $\mathbf{Q H T}^{s}$ (resp. QHT $\left._{=}^{s}\right)$ models. Let $\mathcal{M}=\langle(D, \sigma), H, T\rangle$ be an $\mathcal{L}$-structure that is a model of a universal theory $T$. Then, we call $\mathcal{M}$ a PNA-, UNA-, or SNA-model if the restriction of $\sigma$ to constants in $\mathcal{C}$ is surjective, injective or bijective, respectively.

\subsection{Equilibrium models}

As in the propositional case, quantified equilibrium logic is based on a suitable notion of minimal model.

Definition 6 Among QHT $_{=}^{s}(\mathcal{L})$-structures, we define the order $\unlhd$ as: $\langle(D, \sigma), H, T\rangle \leq$ $\left\{\left(D^{\prime}, \sigma^{\prime}\right), H^{\prime}, T^{\prime}\right\rangle$ if $D=D^{\prime}, \sigma=\sigma^{\prime}, T=T^{\prime}$ and $H \subseteq H^{\prime}$. If the subset relation is strict, we write ' $\triangleleft$ '.

Definition 7 Let $\Gamma$ be a set of sentences and $\mathcal{M}=\langle(D, \sigma), H, T\rangle$ a model of $\Gamma$.

1. $\mathcal{M}$ is said to be total if $H=T$.

2. $\mathcal{M}$ is said to be an equilibrimm model of $\Gamma$ (for short, we say: " $\mathcal{M}$ is in equilibrium") if it is minimal under $\leq$ among models of $\Gamma$, and it is total. 
Notice that a total $\mathbf{Q H T}_{=}^{s}$ model of a theory $\Gamma$ is equivalent to a classical first order model of $\Gamma$.

Proposition 9 Let $\Gamma$ be a theory in $\mathcal{L}$ and $\mathcal{M}$ an equilibrium model of $\Gamma$ in $\mathbf{Q H T}_{=}^{s}\left(\mathcal{L}^{\prime}\right)$ with $\mathcal{L}^{\prime} \supset \mathcal{L}$. Then $\left.\mathcal{M}\right|_{\mathcal{L}}$ is an equilibrium model of $\Gamma$ in $\mathbf{Q H T}_{=}^{s}(\mathcal{L})$.

\subsection{Relation to answer sets}

The above version of QEL is described in more detail in Ref. [27]. If we assume all models are UNA-models, we obtain the version of QEL found in Ref. [26]. There, the relation of QEL to (ordinary) answer sets for logic programs with variables was established (in Ref. [26, Corollary 7.7]). For the present version of QEL, the correspondence can be described as follows.

Proposition 10 [27] Let $\Gamma$ be a universal theory in $\mathcal{L}=\langle C, P\rangle$. Let $\langle U, T, T\rangle$ be a total $\mathbf{Q H T}_{=}^{s}$ model of $\Gamma$. Then $\{U, T, T\rangle$ is an equilibrium model of $\Gamma$ iff $\langle T, T\rangle$ is a propositional equilibrium model of $g r_{U}(\Gamma)$.

By convention, when $\mathcal{P}$ is a logic program with variables we consider the models and equilibrium models of its universal closure expressed as a set of logical formulas. So, from Proposition 10 we obtain:

Corollary 11 Let $\mathcal{P}$ be a logic program. A total $\mathbf{Q H T} \mathbf{T}_{=}^{s}$ model $\{U, T, T\rangle$ of $\mathcal{P}$ is an equilibrium model of $\mathcal{P}$ iff it is a generalised open answer set of $\mathcal{P}$.

Proof It is well-known that for propositional programs equilibrium models coincide with answer sets [24]. Using Proposition 10 and Definition 4 for generalised open answer sets, the result follows.

\section{Relation between hybrid KBs and QEL}

In this section, we show how equilibrium models for hybrid knowledge bases relate to the NM models defined earlier and we show that QEL captures the various approaches to the semantics of hybrid KBs in the literature [16,29-31].

Given a hybrid $\mathrm{KB} K=(\mathcal{T}, \mathcal{P})$, we call $\mathcal{T} \cup \mathcal{P} \cup$ st $(\mathcal{T})$ the stable closute of $\mathcal{K}$, where $s t(\mathcal{T})=\left\{\forall x(p(x) \vee \neg p(x)): p \in \mathcal{L}_{T}\right\}{ }^{10}$ From now on, unless otherwise clear from context, the symbol ${ }^{\prime} \models$ ' denotes the truth relation for $\mathbf{Q H T}{ }_{=}^{s}$. Given a ground program $\mathcal{P}$ and an $\mathcal{L}$-structure $\mathcal{M}=\{U, H, T\}$, the projection $\Pi(\mathcal{P}, \mathcal{M})$ is understood to be defined relative to the component $T$ of $\mathcal{M}$.

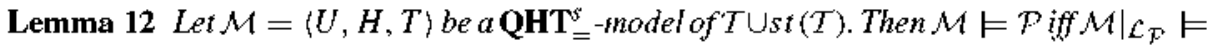
$\Pi\left(g r_{U}(\mathcal{P}), \mathcal{M}\right)$.

Proof By the hypothesis $\mathcal{M} \models\left\{\forall x(p(x) \vee \neg p(x)): p \in \mathcal{L}_{T}\right\}$. It follows that $\left.H\right|_{\mathcal{L}_{T}}=$ $\left.T\right|_{\mathcal{L}_{T}}$. Consider any $r \in \mathcal{P}$, such that $r^{\Pi} \neq \emptyset$. Then, there are four cases to consider. (i) $r$ has the form $\alpha \rightarrow \beta \vee p(t), p(t) \in \mathcal{L}_{T}$ and $p(\sigma(t)) \notin T$, so $\mathcal{M} \models \neg p(t)$. W.l.o.g. assume that $\alpha, \beta \in \mathcal{L}_{P}$, so $r^{\Pi}=\alpha \rightarrow \beta$ and

$$
\left.\mathcal{M} \vDash r \Leftrightarrow \mathcal{M} \models r^{\Pi} \Leftrightarrow \mathcal{M}\right|_{C_{p}} \models r^{\Pi}
$$

${ }^{10}$ Evidently $\mathcal{T}$ becomes stable in $\mathcal{K}$ in the sense that $\forall \varphi \in \mathcal{T}$, st $(\mathcal{T}) \models \neg \neg \varphi \rightarrow \varphi$. The terminology is drawn from intuitionistic logic and mathematics. 
by the semantics for $\mathbf{Q H T}_{=}^{s}$ and Theorem 7. (ii) $r$ has the form $\alpha \rightarrow \beta \vee \neg p(t)$, where $p(\sigma(t)) \in T$; so $p(\sigma(t)) \in H$ and $\mathcal{M} \models p(t)$. Again it is easy to see that (3) holds. Case (iii): $r$ has the form $\alpha \wedge p(t) \rightarrow \beta$ and $p(\sigma(t)) \in H, T$, so $\mathcal{M} \models p(t)$. Case (iv): $r$ has the form $\alpha \wedge \neg p(t) \rightarrow \beta$ and $\mathcal{M} \vDash \neg p(t)$. Clearly for these two cases (3) holds as well. It follows that if $\mathcal{M} \models \mathcal{P}$ then $\left.\mathcal{M}\right|_{\mathcal{L} F} \models \Pi\left(g_{U}(\mathcal{P}), \mathcal{M}\right)$.

To check the converse condition, we need now to only examine the cases where $r^{\Pi}=\emptyset$. Suppose this arises because $p(\sigma(t)) \in H, T$, so $M \models p(t)$. Now, if $p(t)$ is in the head of $r$, clearly $M \vDash r$. Similarly if $\neg p(t)$ is in the body of $r$, by the semantics $M \vDash r$. The cases where $p(\sigma(t)) \notin T$ are analogous and left to the reader. Consequently, if $\left.\mathcal{M}\right|_{\mathcal{L}_{\mathcal{F}}} \vDash$ $\Pi\left(g r_{U}(\mathcal{P}), \mathcal{M}\right)$, then $\mathcal{M} \models \mathcal{P}$.

We now state the relation between equilibrium models and NM-models.

Theorem 13 Let $\mathcal{K}=(\mathcal{T}, \mathcal{P})$ be a hybrid $K B . \mathcal{M}=\langle U, T, T\rangle$ is an equilibrium model of the stable closute of $K$ if and only if $\{U, T\}$ is an NM-model of $K$.

Proof Assume the hypothesis and suppose that $\mathcal{M}$ is in equilibrium. Since $T$ contains only predicates from $\mathcal{L}_{T}$ and $\mathcal{M} \models T \cup$ st $(T)$, evidently

$$
\left.\mathcal{M}\right|_{c_{T}} \models T \cup s t(T)
$$

and so in particular $\left(U,\left.\mathcal{M}\right|_{\mathcal{L}_{T}}\right)$ is a model of $T$. By Lemma 12 ,

$$
\left.\mathcal{M} \vDash \mathcal{P} \Leftrightarrow \mathcal{M}\right|_{\mathcal{L}_{\mathcal{P}}} \vDash \Pi\left(g_{U}(\mathcal{P}), \mathcal{M}\right)
$$

We claim (i) that $\left.\mathcal{M}\right|_{\mathcal{L}_{\mathcal{P}}}$ is an equilibrium model of $\Pi(\mathrm{grv}(\mathcal{P}), \mathcal{M})$. If not, there is a model $\mathcal{M}^{\prime}=\left\langle H^{\prime}, T^{\prime}\right\rangle$ with $H^{\prime} \subset T^{\prime}=\left.T\right|_{\mathcal{L}_{\mathcal{F}}}$ and $\mathcal{M}^{\prime}=\Pi\left(g r_{U}(\mathcal{P}), \mathcal{M}\right)$. Litt $\left(U, \mathcal{M}^{\prime}\right)$ to a (first order) $\mathcal{L}$-structure $\mathcal{N}$ by interpreting each $p \in \mathcal{L}_{T}$ according to $\mathcal{M}$. So $\left.\mathcal{N}\right|_{\mathcal{L}_{T}}=\left.\mathcal{M}\right|_{\mathcal{L}_{T}}$ and by (4) clearly $\mathcal{N} \models T \cup$ st $(\mathcal{T})$. Moreover, by Lemma $12 \mathcal{N} \models \mathcal{P}$ and by assumption $\mathcal{N} \triangleleft \mathcal{M}$, contradicting the assumption that $\mathcal{M}$ is an equilibrium model of $T \cup$ st $(\mathcal{T}) \cup \mathcal{P}$. This establishes (i). Lastly, we note that since $\left\langle\left. T\right|_{\mathcal{C}_{\mathcal{P}}},\left.T\right|_{\mathcal{L}_{\mathrm{F}}}\right\rangle$ is an equilibrium model of $\Pi\left(g r_{U}(\mathcal{P}), \mathcal{M}\right),\left.\mathcal{M}\right|_{\mathcal{L}_{F}}$ is a generalised open answer set of $\Pi\left(\operatorname{gr}_{U}(\mathcal{P}), \mathcal{M}\right)$ by Corollary 11 , so that $\mathcal{M}=\langle U, T, T\rangle$ is an NM-model of $\mathcal{K}$.

For the converse direction, assume the hypothesis but suppose that $\mathcal{M}$ is not in equilibrium. Then, there is a model $\mathcal{M}^{\prime}=\langle U, H, T\rangle$ of $T \cup s t(\mathcal{T}) \cup \mathcal{P}$, with $H \subset T$. Since $\mathcal{M}^{\prime} \models \mathcal{P}$ we can apply Lemma 12 to conclude that $\left.\mathcal{M}^{\prime}\right|_{\mathcal{L}_{P}} \models \Pi\left(\operatorname{gr}_{U}(\mathcal{P}), \mathcal{M}^{\prime}\right)$. But clearly

$$
\Pi\left(g r_{U}(\mathcal{P}), \mathcal{M}^{\prime}\right)=\Pi\left(g r_{U}(\mathcal{P}), \mathcal{M}\right) \text {. }
$$

However, since evidently $\left.\mathcal{M}^{\prime}\right|_{C_{T}}=\left.\mathcal{M}\right|_{\mathcal{C}_{\mathcal{T}}}$, thus $\left.\left.\mathcal{M}^{\prime}\right|_{\mathcal{L}_{\mathrm{F}}} \mathrm{j} \mathcal{M}\right|_{\mathcal{C}_{\mathcal{P}}}$, so this shows that $\left.\mathcal{M}\right|_{\mathcal{C}_{\mathcal{P}}}$ is not an equilibrium model of $\Pi\left(g r_{U}(\mathcal{P}), \mathcal{M}\right)$ and therefore $\left.T\right|_{C_{P}}$ is not an answer set of $\Pi\left(\mathrm{gr}_{U}(\mathcal{P}), \mathcal{M}\right)$ and $\mathcal{M}$ is not an NM- model of $\mathcal{K}$.

This establishes the main theorem relating to the various special types of hybrid $\mathrm{KBs}$ discussed earlier.

Theorem 14 (Main Theorem)

(i) Let $\mathcal{K}^{*}=(\mathcal{T}, \mathcal{P})$ be a g-hybrid (resp. an $r^{+}$-hybrid) knowledge base. $\mathcal{M}=\{U, T, T\}$ is an equilibrium model of the stable closure of $\mathcal{K}$ if and only if $\{U, T\rangle$ is an $N M$-model (resp. $r^{+}-N M$-model) of $\mathcal{K}$.

(ii) Let $\mathcal{K}=(T, \mathcal{P})$ be an r-hybrid knowledge base. Let $\mathcal{M}=\langle U, T, T\rangle$ be an Herbrand model of the stable closure of $\mathcal{K}$. Then $\mathcal{M}$ is in equilibrium in the sense of Ref. [26] if and only if $\{U, T\}$ is an $r$-NM-nodel of $K$. 
Example 5 Consider again the hybrid knowledge base $\mathcal{K}=(\mathcal{T}, \mathcal{P})$, with $\mathcal{T}$ and $\mathcal{P}$ as in Example 1. The stable closure of $\mathcal{K}$, st $(\mathcal{K})=T \cup$ st $(\mathcal{T}) \cup \mathcal{P}$ is

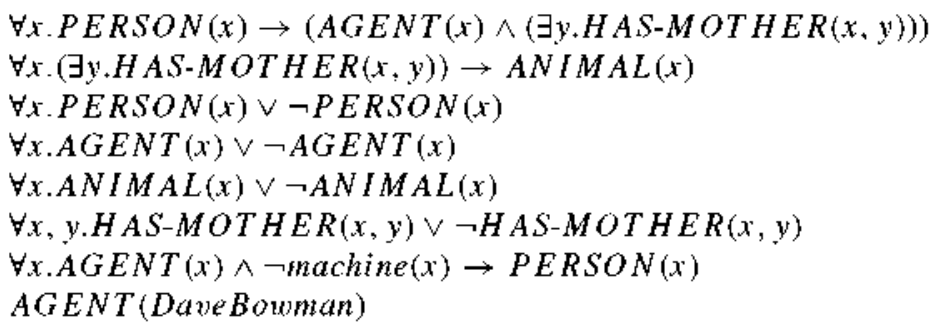

Consider the total HT-model $\mathcal{M}_{H T}=\langle U, I, I\rangle$ of st $(\mathcal{K})$, with $U, I$ as in Example 4. $\mathcal{M}_{H T}$ is not an equilibrium model of $s t(\mathcal{K})$, since $\mathcal{M}_{H T}$ is not minimal among all models: $\left\{U, I^{\prime}, I\right\rangle$, with $I^{\prime}=I \backslash\{$ machine (Dave Bowman) , is a model of $s t(K)$. Furthermore, it is easy to verify that $\left\langle U, I^{\prime}, I^{\prime}\right\rangle$ is not a model of $s t(\mathcal{K})$.

Now, consider the total HT-model $\mathcal{M}_{H T}^{\prime}=\langle U, M, M\rangle$, with $U$ as before, and

$M=\{A G E N T($ DaveBowman $), P E R S O N($ DaveBowman $)$,

ANIMAL(DaveBowman), HAS - NAME(DaveBowman, $k)\}$.

$\mathcal{M}_{H T}^{\prime}$ is an equilibrium model of $s t(\mathcal{K})$. Indeed, consider any $M^{\prime} \subset M$. It is easy to verify that $\left\langle U, M^{\prime}, M\right\rangle$ is not a model of $s t(\mathcal{K}) . \diamond$

\section{Discussion}

We have seen that quantified equilibrium logic captures three of the main approaches to integrating classical, first-order or DL knowledge bases with nonmonotonic rules under the answer set semantics, in a modular, hybrid approach. However, QEL has a quite distinct flavour from those of $\mathrm{r}$-hybrid, $\mathrm{r}^{+}$-hybrid and g-hybrid KBs. Each of these hybrid approaches has a semantics composed of two different components: a classical model on the one hand and an answer set on the other. Integration is achieved by the fact that the classical model serves as a pre-processing tool for the rule base. The style of QEL is different. There is one semantics and one kind of model that covers both types of knowledge. There is no need for any pre-processing of the rule base. In this sense, the integration is more far-reaching. The only distinction we make is that for that part of the knowledge base considered to be classical and monotonic we add a stability condition to obtain the intended interpretation.

There are other features of the approach using QEL that are worth highlighting. First, it is based on a simple minimal model semantics in a known nonclassical logic, actually a quantified version of Gödel's 3-valued logic. No reducts are involved and, consequently, the equilibrium construction applies directly to arbitrary first-order theories. The rule part $\mathcal{P}$ of a knowledge base might therefore comprise, say, a nested logic program, where the heads and bodies of rules may be arbitrary boolean formulas, or perhaps rules permitting nestings of the implication connective. While answer sets have recently been defined for such general formulas, more work would be needed to provide integration in a hybid $\mathrm{KB}$ setting. ${ }^{11}$ Evidently, QEL in the general case is undecidable, so for extensions of the rule language syotax for practical applications one may wish to study restrictions analogous to safety or

11 For a recent extension of answer sets to fitst-order formulas, see [12], which is explained in more detail in Sect. 8. 
guardedness. Second, the logic $\mathbf{Q H T}_{=}^{s}$ can be applied to characterise properties such as the strong equivalence of programs and theories [20,27]. While strong equivalence and related concepts have been much studied recently in ASP, their characterisation in the case of hybrid KBs remains uncharted territory. The fact that QEL provides a single semantics for hybrid KBs means that a simple concept of strong equivalence is applicable to such KBs and characterisable using the underlying logic, $\mathbf{Q H T}{ }_{=}^{s}$. In Sect. 9 below, we describe how $\mathbf{Q H T}_{=}^{s}$ can be applied in this context.

\section{Hybrid KBs and the SM operator}

Recently, Ferraris, Lee and Lifschitz [12] have presented a new definition of stable models. It is applicable to sentences or finitely axiomatisable theories in first-order logic. The definition is syntactical and involves an operator SM that resembles parallel circumscription. The stable models of a sentence $F$ are the structures that satisfy a certain second-order sentence, $\mathrm{SM}[F]$. This new definition of stable model agrees with equilibrium logic in the sense that the models of SM $[F]$ from [12] are essentially the equilibrium models of $F$ as defined in this article.

We shall now show that by slightly modifying the SM operator, we can also capture the NM semantics of hybrid knowledge bases. First, we need to introduce some notation, essentially following [20].

If $p$ and $q$ are predicate constants of the same arity then $p=q$ stands for the formula

$$
\forall \mathbf{x}(p(\mathbf{x}) \leftrightarrow q(\mathbf{x})),
$$

and $p \leq q$ stands for

$$
\forall \mathbf{x}(p(\mathbf{x}) \rightarrow q(\mathbf{x}))
$$

where $\mathbf{x}$ is a tuple of distinct object variables. If $\mathbf{p}$ and $\mathbf{q}$ are tuples $p_{1}, \ldots, p_{n}$ and $q_{1}, \ldots, q_{n}$ of predicate constants then $\mathbf{p}=\mathbf{q}$ stands for the conjunction

$$
p_{1}=q_{1} \wedge \cdots \wedge p_{n}=q_{n}
$$

and $\mathbf{p} \leq \mathbf{q}$ for

$$
p_{1} \leq q_{1} \wedge \cdots \wedge p_{n} \leq q_{n}
$$

Finally, $\mathbf{p}<\mathbf{q}$ is an abbreviation for $\mathbf{p} \leq \mathbf{q} \wedge \neg(\mathbf{p}=\mathbf{q})$. The operator $\left.N M\right|_{p}$ defines second-order formulas and the previous notation can be also applied to tuples of predicate variables.

$$
\left.\mathrm{NM}\right|_{\mathcal{P}}[F]=F \wedge \neg \exists \mathbf{u}\left((\mathbf{u}<\mathbf{p}) \wedge F^{*}(\mathbf{u})\right),
$$

where $\mathbf{p}$ is the list of all predicate constants $p_{1}, \ldots, p_{n} \notin \mathcal{C}_{T}$ occurring in $F$, $\mathbf{u}$ is a list of $n$ distinct predicate variables $u_{1}, \ldots, u_{n}$. The $\left.\mathrm{NM}\right|_{p}$ operator works just like in the SM operator from Ref. [12] except that the substitution of predicates $p_{\mathrm{i}}$ is restricted to those not in $\mathcal{L}_{T}$. Notice that in the definition of $\mathrm{NM} \mid p[F]$ the second conjunct specifies the minimality condition on interpretations while the third conjunct involves a translation ${ }^{*+*}$ that provides a reduction of the nonclassical here-and-there logic to classical logic. This translation is recursively defined as follows: 
$-p_{i}\left(t_{1}, \ldots, t_{m}\right)^{*}=u_{i}\left(t_{1}, \ldots, t_{m}\right)$ if $p_{i} \notin \mathcal{L}_{T}$;

$-p_{i}\left(t_{1}, \ldots, t_{m}\right)^{*}=p_{i}\left(t_{1}, \ldots, t_{m}\right)$ if $p_{i} \in \mathcal{L}_{T}$;

$-\left(t_{1}=t_{2}\right)^{*}=\left(t_{1}=t_{2}\right)$;

$-\quad \perp^{*}=\perp$;

- $(F \odot G)^{*}=F^{*} \odot G^{*}$, where $\odot \in\{\wedge, \vee\}$;

- $(F \rightarrow G)^{*}=\left(F^{*} \rightarrow G^{*}\right) \wedge(F \rightarrow G)$

- $(Q x F)^{*}=Q x F^{*}$, where $Q \in\{\forall, \exists\}$.

(There is no clause for negation here, because $\neg F$ is treated as shorthand for $F \rightarrow \perp$.)

Theorem $15 \mathcal{M}=\langle U, T\rangle$ is a NM-model of $\mathcal{K}=(\mathcal{T}, \mathcal{P})$ if and only if it satisfies $T$ and $\mathrm{NM} \mid \mathcal{P}[\mathcal{P}]$.

We assume here that both $\mathcal{T}$ and $\mathcal{P}$ are finite, so that the operator $\left.\mathrm{NM}\right|_{\mathcal{P}}$ is well-defined.

Proof $(\Rightarrow)$ If $\{U, T\}, U=(D, \sigma)$, is a NM-model of $\mathcal{K}=\langle\mathcal{T}, \mathcal{P})$, then $\langle U, T\rangle \models \mathcal{T}$, and $\langle U, T\rangle \models \mathcal{P}$, and $\langle U, T, T\rangle$ is an equilibrium model of $\mathcal{T} \cup$ st $(\mathcal{T}) \cup \mathcal{P}$. So we only need to prove that $\{U, T\rangle \models-\exists \mathbf{u}\left((\mathbf{u}<\mathbf{p}) \wedge P^{*}(\mathbf{u})\right)$. For the contradiction, let us assume that

$$
\langle U, T\rangle \models \exists \mathbf{u}\left((\mathbf{u}<\mathbf{p}) \wedge \mathcal{P}^{*}(\mathbf{u})\right)
$$

This means that:

Fact 1: For every $p_{i} \notin \mathcal{L}_{T}$, there exists $\bar{p}_{i} \subset D^{n}$ such that $(\mathbf{u}<\mathbf{p}) \wedge \mathcal{P}^{*}(\mathbf{u})$ is valid in the structure $\langle U, T\rangle$ where $u_{i}$ is interpreted as $\bar{p}_{i}$.

If we consider the set

$$
\begin{aligned}
H= & \left\{p_{i}\left(d_{1}, \ldots, d_{k}\right):\left(d_{1}, \ldots, d_{k}\right) \in \bar{p}_{i}\right\} \cup \\
& \cup\left\{p_{i}\left(d_{1}, \ldots, d_{k}\right): p_{i} \in \mathcal{L}_{T}, p_{i}\left(d_{1}, \ldots, d_{k}\right) \in T\right\},
\end{aligned}
$$

and $u_{i}$ is interpreted as $\bar{p}_{i}$, then $\mathbf{u}<\mathbf{p}$ is valid in $\langle U, T\rangle$ iff $H \subset T$ and $\mathcal{P}^{*}(\mathbf{u})$ is valid in $\langle U, T\rangle$ iff $\langle U, H\rangle \vDash \mathcal{P}^{*}(\mathbf{p})$; that is, the Fact 1 is equivalent to:

$$
H \subset T \text { and } \quad\langle U, H\rangle \models \mathcal{P}^{*}(\mathbf{p})
$$

Since $T 、 H$ does not include predicate symbols of $\mathcal{C}_{T},\langle U, H, T\rangle \models \mathcal{T} \cup$ st $(\mathcal{T})$. So, to finish the proof, we need to prove the following for every formula $\varphi$ :

Fact 2: $\{U, H, T\}, h \models \varphi$ if and only if $\{U, H\rangle \models \varphi^{*}(\mathbf{p})$.

As a consequence of Fact 2, we have that $\langle U, H, T\rangle \models \mathcal{P}$ and thus $\langle U, H, T\rangle$ is a model of the stable closure of $\mathcal{K}$, which contradicts that $\{U, T, T\rangle$ is in equilibrium. Fact 2 is proved by induction on $\varphi$ :

(i) If $\varphi=p_{i}\left(d_{1}, \ldots, d_{k}\right)$, then $\varphi^{*}(\mathbf{p})=\varphi$ :

$$
\langle U, H, T\}, h \models \psi \Leftrightarrow p_{i}\left(d_{1}, \ldots, d_{k}\right) \in H \Leftrightarrow\langle U, H\rangle \models \varphi^{*}(\mathbf{p})
$$

(ii) Let $\psi=\psi_{1} \wedge \psi_{2}$ and assume that, for $i=1,2$,

$$
\langle U, H, T\}, h \models \psi_{i} \quad \text { iff }\langle U, H\rangle \models \psi_{i}^{*}(\mathbf{p}) .
$$

* For $\varphi=\psi_{1} \wedge \psi_{2}$ under assumption (7):

$$
\begin{aligned}
\langle U, H, T\rangle, h \models \psi_{1} \wedge \psi_{2} & \Leftrightarrow\langle U, H, T\rangle, h \models \psi_{1} \text { and }\langle U, H, T\rangle, h \models \psi_{2} \\
& \Leftrightarrow\langle U, H\rangle \models \psi_{1}^{*}(\mathbf{p}) \text { and }\langle U, H\rangle \models \psi_{2}^{*}(\mathbf{p}) \\
& \Leftrightarrow\langle U, H\rangle \models\left(\psi_{1} \wedge \psi_{2}\right)^{*}
\end{aligned}
$$


* Similarly, for $\varphi=\psi_{1} \rightarrow \psi_{2}$ under assumption (7):

$$
\begin{aligned}
& \langle U, H, T\rangle, h \models \psi_{1} \rightarrow \psi_{2} \Leftrightarrow \\
& \Leftrightarrow\{U, H, T\rangle, t \models \psi_{1} \rightarrow \psi_{2} \text { and } \\
& \quad \text { either }\langle U, H, T\rangle, h \models \psi_{1} \text { or }\langle U, H, T\rangle, h \models \psi_{2} \\
& \Leftrightarrow\langle U, T\rangle \models \psi_{1} \rightarrow \psi_{2} \text { and either }\langle U, H\rangle \models \psi_{1}^{*}(\mathbf{p}) \text { or }\langle U, H\rangle \models \psi_{2}^{*}(\mathbf{p}) \\
& \Leftrightarrow\langle U, H\rangle \models\left(\psi_{1} \rightarrow \psi_{2}\right)^{*}
\end{aligned}
$$

$(\Leftarrow) \quad$ If $\langle U, T\}, U=(D, \sigma)$, satisfies $T$ and $\left.\mathrm{NM}\right|_{\mathcal{P}}[\mathcal{P}]$, then trivially $\langle U, T, T\rangle$ is a here-and-there model of the closure of $\mathcal{K}$; we only need to prove that this model is in equilibrium. By contradiction, let us assume that $\langle U, H, T\rangle$ is a here-and-there model of the closure of $\mathcal{K}$ with $H \subset T$. For every $p_{i} \notin \mathcal{L}_{T}$, we define

$$
\bar{p}_{i}=\left\{\left(d_{i}, \ldots, d_{k}\right): p_{i}\left(d_{i}, \ldots, d_{k}\right) \in H\right\}
$$

Fact 3: $(\mathbf{u}<\mathbf{p}) \wedge \mathcal{P}^{*}(\mathbf{u})$ is valid in the structure $\{U, T\}$ if the variables $u_{i}$ are interpreted as $\bar{p}_{i}$.

As a consequence of Fact 3, we have that $\exists \mathbf{u}\left((\mathbf{u}<\mathbf{p}) \wedge \mathcal{P}^{*}(\mathbf{u})\right)$ is satisfied by $\{U, T\}$ which contradicts that $\left.\mathrm{NM}\right|_{\mathcal{P}}[\mathcal{P}]$ is satisfied by the structure.

As in the previous item, Fact 3 is equivalent to

$$
H \subset T \text { and } \quad\{U, H\rangle \models \mathcal{P}^{*}(\mathbf{p})
$$

The first condition, $H \subset T$, is trivial by definition and the second one is a consequence of Fact 2 .

\section{The strong equivalence of knowledge bases}

Let us see how the previous results, notably Theorem 13, can be applied to characterise a concept of strong equivalence between hybrid knowledge bases. It is important to know when different reconstructions of a given body of knowledge or state of affairs are equivalent and lead to essentially the same problem solutions. In the case of knowledge reconstructed in classical logic, ordinary logical equivalence can serve as a suitable concept when applied to theories formulated in the same vocabulary. In the case where nonmonotonic rules are present, however, the situation changes: two sets of rules may have the same answer sets yet behave very differently once they are embedded in some larger context. Thus for hybrid knowledge bases, one may also like to know that equivalence is robust or modular. A robust notion of equivalence for logic programs will require that programs behave similarly when extended by any further programs. This leads to the following concept of strong equivalence: programs $\Pi_{1}$ and $\Pi_{2}$ are strongly equivalent if and only if for any set of rules $\Sigma, \Pi_{1} \cup \Sigma$ and $\Pi_{2} \cup \Sigma$ have the same answer sets. This concept of strong equivalence for logic programs in ASP was introduced and studied in Ref. [19] and has given rise to a substantial body of further work looking at different characterisations, new variations and applications of the idea, as well as the development of systems to test for strong equivalence. Strong equivalence has also been defined and studied for logic programs with variables and first-order nonmonotonic theories under the stable model or equilibrium logic semantics $[8,20,22,27]$. In equilibrium logic we say that two (first-order) theories $\Pi_{1}$ and $\Pi_{2}$ are strongly equivalent if and only if for any theory $\Sigma, \Pi_{1} \cup \Sigma$ and $\Pi_{2} \cup \Sigma$ have the same equilibrium models [20,27]. Under this definition we have: 
Theorem 16 [20,27] Two (first-order) theories $\Pi_{1}$ and $\Pi_{2}$ are strongly equivalent if and only if they are equivalent in $\mathbf{Q H T}_{=}^{s}$.

Different proofs of Theorem 16 are given in Refs. [20] and [27]. For present purposes, the proof contained in Ref. [27] is more useful. It shows that if theories are not strongly equivalent, the set of formulas $\Sigma$ such that $\Pi_{1} \cup \Sigma$ and $\Pi_{2} \cup \Sigma$ do not have the same equilibrium models can be chosen to have the form of implications $(A \rightarrow B)$ where $A$ and $B$ are atomic. So if we are interested in the case where $\Pi_{1}$ and $\Pi_{2}$ are sets of rules, $\Sigma$ can also be regarded as a set of rules. We shall make use of this property below.

In the case of hybrid knowledge bases $\mathcal{K}=(T, \mathcal{P})$, various kinds of equivalence can be specified, according to whether one or other or both of the components $T$ and $\mathcal{P}$ are allowed to vary. The following form is rather general.

Definition 8 Let $\mathcal{K}_{1}=\left(\mathcal{T}_{1}, \mathcal{P}_{1}\right)$ and $\mathcal{K}_{2}=\left(\mathcal{T}_{2}, \mathcal{P}_{2}\right)$, be two hybrid $\mathrm{KB}$ s sharing the same structural language, ie. $\mathcal{L}_{T_{1}}=\mathcal{L}_{T_{2}}, \mathcal{K}_{1}$ and $\mathcal{K}_{2}$ are said to be strongly equivalent if for any theory $\mathcal{T}$ and set of rules $\mathcal{P},\left(\mathcal{T}_{1} \cup \mathcal{T}, \mathcal{P}_{1} \cup \mathcal{P}\right)$ and $\left(\mathcal{T}_{2} \cup \mathcal{T}, \mathcal{P}_{2} \cup \mathcal{P}\right)$ have the same NM-models.

Until further notice, let us suppose that $\mathcal{K}_{1}=\left(\mathcal{T}_{1}, \mathcal{P}_{1}\right)$ and $\mathcal{K}_{2}=\left(\mathcal{T}_{2}, \mathcal{P}_{2}\right)$ are hybrid $\mathrm{KBs}$ sharing a common structural language $\mathcal{L}$.

Proposition $17 \mathcal{K}_{1}$ and $\mathcal{K}_{2}$ are strongly equivalent if and only if $\mathcal{T}_{1} \cup$ st $\left(\mathcal{T}_{1}\right) \cup \mathcal{P}_{1}$ and $\mathcal{T}_{2} \cup$ st $\left(\mathcal{T}_{2}\right) \cup \mathcal{P}_{2}$ are logically equivalent in $\mathbf{Q H} \mathbf{T}_{=}^{s}$.

Proof Let $\mathcal{K}_{1}=\left(\mathcal{T}_{1}, \mathcal{P}_{1}\right)$ and $\mathcal{K}_{2}=\left(\mathcal{T}_{2}, \mathcal{P}_{2}\right)$ be hybrid KBs such that $\mathcal{L}_{T_{1}}=\mathcal{L}_{T_{2}}=\mathcal{L}$. Suppose $\mathcal{T}_{1} \cup$ st $\left(\mathcal{T}_{1}\right) \cup \mathcal{P}_{1}$ and $\mathcal{T}_{2} \cup$ st $\left(\mathcal{T}_{2}\right) \cup \mathcal{P}_{2}$ are logically equivalent in $\mathbf{Q H T} \mathbf{P}_{=}^{s}$. Clearly $\left(\mathcal{T}_{1} \cup \mathcal{T} \cup\right.$ st $\left.\left(\mathcal{T}_{1} \cup \mathcal{T}\right) \cup \mathcal{P}_{1} \cup \mathcal{P}\right)$ and $\left(\mathcal{T}_{2} \cup \mathcal{T} \cup \operatorname{st}\left(\mathcal{T}_{2} \cup \mathcal{T}\right) \cup \mathcal{P}_{2} \cup \mathcal{P}\right)$ have the same QHT $_{=}^{s}$-models and hence the same equilibrium models. Strong equivalence of $\mathcal{K}_{1}$ and $\mathcal{K}_{2}$ follows by Theorem 13.

For the 'only-if' direction, suppose that $\mathcal{T}_{1} \cup s t\left(\mathcal{T}_{1}\right) \cup \mathcal{P}_{1}$ and $\mathcal{T}_{2} \cup$ st $\left(\mathcal{T}_{2}\right) \cup \mathcal{P}_{2}$ are not logically equivalent in $\mathbf{Q H T}_{=}^{s}$, so there is.an $\mathbf{Q H T}{ }_{=}^{s}$-model of one of these theories that is not an $\mathbf{Q H T}^{s}$ of the other. Applying the proof of Theorem 16 given in Ref. [27] we can infer that there is a set $\mathcal{P}$ of rules of a simple sort such that the equilibrium models of $\mathcal{T}_{1} \cup$ st $\left(\mathcal{T}_{1}\right) \cup \mathcal{P}_{1} \cup \mathcal{P}$ and $\mathcal{T}_{2} \cup s t\left(\mathcal{T}_{2}\right) \cup \mathcal{P}_{2} \cup \mathcal{P}$ do not coincide. Hence by Theorem $13 \mathcal{K}_{1}$ and $\mathcal{K}_{2}$ are not strongly equivalent.

Notice that from the proof of Proposition 17, it follows that the nonstrong equivalence of two hybrid knowledge bases can always be made manifest by choosing extensions having a simple form, obtained by adding simple rules to the rule base.

We mention some conditions to test for strong equivalence and nonequivalence.

Corollary 18 (a) $\mathcal{K}_{1}$ and $\mathcal{K}_{2}$ are strongly equivalent if $\mathcal{T}_{1}$ and $\mathcal{T}_{2}$ are classically equivalent and $\mathcal{P}_{1}$ and $\mathcal{P}_{2}$ are equivalent in $\mathbf{Q H T} \mathbf{T}_{=}^{s}$.

(b) $\mathcal{K}_{1}$ and $\mathcal{K}_{2}$ are not strongly equivalent if $\mathcal{T}_{1} \cup \mathcal{P}_{1}$ and $\mathcal{T}_{2} \cup \mathcal{P}_{2}$ are not equivalent in classical logic.

Proof (a) Assume the hypothesis. Since $\mathcal{K}_{1}=\left(\mathcal{T}_{1}, \mathcal{P}_{1}\right)$ and $\mathcal{K}_{2}=\left(\mathcal{T}_{2}, \mathcal{P}_{2}\right)$ share a common structural language $\mathcal{L}$, it follows that $\operatorname{si}\left(\mathcal{T}_{1}\right)=s t\left(\mathcal{T}_{2}\right)=\mathcal{S}$, say. Since $\mathcal{T}_{1}$ and $\mathcal{T}_{2}$ are classically equivalent, $\mathcal{T}_{1} \cup \mathcal{S}$ and $\mathcal{T}_{2} \cup \mathcal{S}$ have the same (total) $Q H T_{=}^{s}$-models and so for any $\mathcal{T}$ also $\mathcal{T}_{1} \cup \mathcal{T} \cup \mathcal{S} \cup \operatorname{st}(\mathcal{T})$ and $\mathcal{T}_{2} \cup \mathcal{T} \cup \mathcal{S} \cup$ st $(\mathcal{T})$ have the same (total) $\mathbf{Q H T}{ }^{s}$-models. Since $\mathcal{P}_{1}$ and $\mathcal{P}_{2}$ are equivalent in $Q \mathbf{Q H T} \mathbf{T}_{=}^{s}$ it follows also that for any $\mathcal{P},\left(T_{1} \cup \mathcal{T} \cup \mathcal{S} \cup\right.$ st $\left.(\mathcal{T}) \cup \mathcal{P}_{1} \cup \mathcal{P}\right)$ and $\left(T_{2} \cup \mathcal{T} \cup \mathcal{S} \cup s t(\mathcal{T}) \cup \bar{P}_{2} \cup \mathcal{P}\right)$ have the same $\mathbf{Q H T}{ }^{s}$-models and hence the same equilibrium models. The conclusion follows by Theorem 13. 
(b) Suppose that $T_{1} \cup \mathcal{P}_{1}$ and $T_{2} \cup \mathcal{P}_{2}$ are not equivalent in classical logic. Assume again that $s t\left(T_{1}\right)=s t\left(\mathcal{T}_{2}\right)=\mathcal{S}$, say. Then clearly $\mathcal{T}_{1} \cup \mathcal{S} \cup \mathcal{P}_{1}$ and $\mathcal{T}_{2} \cup \mathcal{S} \cup \mathcal{P}_{2}$ are not classically equivalent and hence they cannot be $Q H_{T}^{*}=$-equivalent. Applying the second part of the proof of Proposition 17 completes the argument.

Special cases of strong equivalence arise when hybrid KBs are based on the same classical theory, say, or share the same rule base. That is, $\left(\mathcal{T}, \mathcal{P}_{1}\right)$ and $\left(\mathcal{T}, \mathcal{P}_{2}\right)$ are strongly equivalent if $\mathcal{P}_{1}$ and $\mathcal{P}_{2}$ are $\mathbf{Q H T}^{s}$-equivalent. ${ }^{12}$ Analogously:

\section{$\left(T_{1}, \mathcal{P}\right)$ and $\left(T_{2}, \mathcal{P}\right)$ are strongly equivalent if $\mathcal{T}_{1}$ and $T_{2}$ are classically equivalent.}

Let us briefly comment on a restriction that we imposed on strong equivalence, namely that the KBs in question share a common structural language. Intuitively, the reason for this is that the structural language $\mathcal{L}_{T}$ associated with a hybrid knowledge base $\mathcal{K}=(\mathcal{T}, \mathcal{P})$ is part of its identity or 'meaning'. Precisely the predicates in $\mathcal{L}_{T}$ are the ones treated classically. In fact, another $\mathrm{KB}, K^{\prime}=\left(\mathcal{T}^{\prime}, \mathcal{P}\right)$, where $\mathcal{T}^{\prime}$ is completely equivalent to $\mathcal{T}$ in classical logic, may have a different semantics if $\mathcal{L}_{T}$ is different from $\mathcal{L}_{T}$. To see this, let us consider a simple example in propositional logic. Let $\mathcal{K}_{1}=\left(\mathcal{T}_{1}, \mathcal{P}_{1}\right)$ and $\mathcal{K}_{2}=\left(\mathcal{T}_{2}, \mathcal{P}_{2}\right)$, be two hybrid KBs where $\mathcal{P}_{1}=\mathcal{P}_{2}=\{(p \rightarrow q)\}, \mathcal{T}_{1}=\{(r \wedge(r \vee p))\}, \mathcal{T}_{2}=\{r\}$. Clearly, $\mathcal{T}_{1}$ and $\mathcal{T}_{2}$ are classically and even $\mathbf{Q H T} \mathbf{T}_{=}^{s}$-equivalent. However, $\mathcal{K}_{1}$ and $\mathcal{K}_{2}$ are not even in a weak sense semantically equivalent. $s t\left(\mathcal{T}_{1}\right)=\{r \vee \neg r ; p \vee \neg p\}$, while $s t\left(\mathcal{T}_{2}\right)=\{r \vee \neg r\}$. It is easy to check that $\mathcal{T}_{1} \cup s t\left(\mathcal{T}_{1}\right) \cup \mathcal{P}_{1}$ and $\mathcal{T}_{2} \cup s t\left(\mathcal{T}_{2}\right) \cup \mathcal{P}_{2}$ have different $\mathbf{Q H T}{ }^{s}$-models, different equilibrium models and (hence) $K_{1}$ and $K_{2}$ have different NM-models. So we see that without the assumption of a common structural language, the natural properties expressed in Corollary 18 (a) and (8) would no longer hold.

It is interesting to note here that meaning-preserving relations among ontologies have recently become a topic of interest in the description logic community where logical concepts such as that of conservative extension are currently being studied and applied [13]. A unified, logical approach to hybrid KBs such as that developed here should lend itself well to the application of such concepts.

\section{Related work and conclusions}

We have provided a general notion of hybrid knowledge base, combining first-order theories with nonmonotonic rules, with the aim of comparing and contrasting some of the different variants of hybrid $\mathrm{KB}$ s found in the literature [16,29-31]. We presented a version of quantified equilibrium logic, $Q E L$, without the unique names assumption, as a unified logical foundation for hybrid knowledge bases. We showed how for a hybrid knowledge base $\mathcal{K}$ there is a natural correspondence between the nonmonotonic models of $\mathcal{K}$ and the equilibrium models of what we call the stable closure of $\mathcal{K}$. This yields a way to capture in QEL the semantics of the g-hybrid KBs of Heymans et al. [16] and the r-hybrid KBs of Rosati [30], where the latter is defined without the UNA but for safe programs. Similarly, the version of QEL with UNA captures the semantics of r-hybrid KBs as defined in Ref. [29,31]. It is important to note that the aim of this paper was not that of providing new kinds of safety conditions or decidability results; these issues are ably dealt with in the literature reviewed here. Rather our objective has been to show how classical and nonmonotonic theories might be unified under a single semantical model. In part, as [16] show with their reduction of DL knowledge bases

$\overline{12}$ In Ref. [5] it was incorrectly stated in Proposition 7 that this condition was both necessary and sufficient for strong equivalence, instead of merely sufficient. 
to open answer set programs, this can also be achieved (at some cost of translation) in other approaches. What distinguishes QEL is the fact that it is based on a standard, nonclassical logic, $\mathbf{Q H T}_{=}^{s}$, which can therefore provide a unified logical foundation for such extensions of (open) ASP. To illustrate the usefulness of our framework we showed how the logic QHT= also captures a natural concept of strong equivalence between hybrid knowledge bases.

There are several other approaches to combining languages for Ontologies with nonmonotonic rules which can be divided into two main streams [3]: approaches which define integration of rules and ontologies (a) by entailment, ie. querying classical knowledge bases through special predicates the rules body, and (b) on the basis of single models, ie. defining a common notion of combined model.

The most prominent of the former kind of approaches are dl-programs [10] and their generalisation, HEX-programs [9]. Although these approaches both are based on ASP like our approach, the orthogonal view of integration by entailment can probably not be captured by a simple embedding in QEL. Another such approach which allows querying classical KBs from a nonmonotonic rules language is based on Defeasible Logic [33].

As for the second stream, variants of Autoepistemic Logic [4], and the logic of minimal knowledge and negation as failure (MKNF) [23] have been recently proposed in the literature. Similar to our approach, both these approaches embed a combined knowledge base in a unifying logic. However, both purchase use modal logics fact syntactically and semantically extend first-order logics. Thus, in these approaches, embedding of the classical part of the theory is trivial, whereas the nonmonotonic rules part needs to be rewritten in terms of modal formulas. Our approach is orthogonal, as we use a nonclassical logic where the nonmonotonic rules are trivially embedded, but the stable closure guarantees classical behaviour of certain predicates. In addition, the fact that we include the stable closure ensures that the predicates from the classical parts of the theory behave classically, also when used in rules with negation. In contrast, in both modal approaches occurrences of classical predicates are not interpreted classically, as illustrated in the following example.

Example 6 Consider the theory $\mathcal{T}=\{A(a)\}$ and the program $\mathcal{P}=\{r \leftarrow \neg A(b)\}$. We have that there exists an NM-model $\mathcal{M}$ of $(\mathcal{T}, \mathcal{P})$ such that $\mathcal{M} \vDash A(a), A(b)$ and $\mathcal{M} \forall$ $A(a), A(b)$, and so $r$ is not entailed. Consider now the embedding $\tau_{H P}$ of logic programs into autoepistemic logic [4]. We have $\tau_{H P}(\mathcal{P})=\{\neg \mathrm{L} A(b) \rightarrow r\}$. In autoepistemic logic, $\mathrm{L} A(b)$ is true iff $A(b)$ is included in a stable expansion $T$, which is essentially the set of all entailed formulas, assuming $T$. We have that $A(b)$ is not entailed from $T \cup \tau_{H P}(\mathcal{P})$ under any stable expansion, and so $\mathrm{L} A(b)$ is false, and thus $r$ is necessarily true in every model. We thus have that $r$ is a consequence of $\mathcal{T} \cup \tau_{H P}(\mathcal{P})$.

Similar for the hybrid MKNF knowledge bases by Motik and Rosati [23].

As shown by Bruijo et al. [6], adding classical interpretation axioms-essentially a modal version of the stable closure axioms - to the theory $T \cup \tau_{H P}(\mathcal{P})$ allows one to capture the hybrid knowledge base semantics we considered in this paper.

In future work, we hope to consider further aspects of applying QEL to the domain of hybrid knowledge systems. Extending the language with functions symbols and with strong negation is a routine task, since QEL includes these items already. We also plan to consider in the future how QEL can be used to define a catalogue of logical relations between hybrid KBs. Last, but not least, let us mention that in this paper we exclusively dealt with hybrid combinations of classical theories with logic programs under variants of the stable-model semantics. Recently, also hybrid rule combinations based on the well-founded semantics have been proposed by Drabent et al. [7] or Knorr et al. [18], defining an analogous, modular 
semantics like hybrid knowledge bases considered here. In this context, we plan to investigate whether a first-order version of Partial Equilibrium Logic [2], which has been recently shown to capture the well-founded semantics in the propositional case, can similarly work as a foundation for hybrid rule combinations à la Drabent et al. [7].

We believe that on the long run the general problem addressed by the theoretical foundations layed in this paper could potentially provide essential insights for realistic applications of ontologies and Semantic Web technologies in general, since for most of these applications current classical ontology languages provide too limited expressivity and the addition of nonmonotonic rules is key to overcome these limitations. As an example, let us mention the "clash" between the open world assumption in ontologies and the nonmonotonicity/closed world nature of typical Semantic Web query languages such as SPARQL, which contains nonmonotonic constructs and in fact can be translated to rules with nonmonotonic negation [28]. While some initial works exist in the direction of using SPARQL on top of OWL [17], the foundations and exact semantic treatment of corner cases is still an open problem. ${ }^{13}$ As another example, let us mention mappings between modular ontologies as for instance investigated by Ensan [11]; nonmonotonic rules could provide a powerful tool to describe mappings between ontologies.

Acknowledgments Part of the tesults in this paper are contained, in preliminary form, in the proceedings of the 1st International Conference on Web Reasoning and Rule Systems (RR2007), and in the informal proceedings of the RuleML-06 Workshop on Ontology and Rule Integration. The authors thank the anonymous reviewers of those preliminary versions of the article for their helpful comments. This research has been partially supported by the Spanish MEC (now MCI) under the projects TIC-2003-9001. TIN2006-15455CO3, and CSD2007-00022, also by the project URJC-CM-2006-CET-0300 and by the European Commission undet the projects Knowledge Web (IST-2004-507482) and inContext (IST-034718), as well as by Science Fonndation Ireland under Grant No. SFI/08/CE/1380 (Lion-2).

\section{References}

1. Baral C (2002) Knowledge representation, reasoning and declarative problem solving. Canbridge University Press. Cambridge

2. Cabalat P, Odintsov SP. Pearce D, Valverde A (2006) Analysing and extending well-fonted and partial stable semantics using partial eguilibrium logic. In: Proceedings of the 22 nd international conference on logic progranming (ICLP 2006), vol 4079 of lecture notes in computer science. Springer, Seattle, WA. USA. pp 346-360

3. de Brujjn J, Eiter T, Polleres A. Tonjpits H (2006) On representational issues about conbinations of classical theories with nonmonotonic rules. In: Proceedings of the fitst international conference on knowledge science, Engineering and management (KSEM'06), number 4092 in lecture notes in computer science, Springer-Verlag, Guilin, China

4. de Brujin J. Eiter T, Polleres A, Tompits H (2007) Enbedding non-ground logic programs into autoepistemic logic for knowledge-base combination. In: Proceedings of the twentieth international joint conference on artificial intelligence (IJCAI-07), AAAI, Hyderabad, India, pp 304-309

5. de Bruijn J. Pearce D, Polleres A, Valverde A (2007) Quantified equilibrium logic and hybrid rules, In: First international conference on web reasoning and rule systems (RR2007), vol 4524 of lecture notes in computer science. Springer, Innsbiuck, Austria, pp 58-72

6. de Biujjn J. Eiter T. Tompits H (2008) Embedding approaches to combining rules and ontologies into autoepistemic logic. In: Proceedings of the 11 th international conference on ptinciples of knowledge representation and reasoning (KR2008), AAAI, Sydney, Australia, pp 485-495

13 One of the authors of the present paper is in fact chairing the W3C SPARQL working group in which at the time of writing of this paper this topic is being discussed actively. 
7. Drabent W, Henriksson J, Maluszynski J (2007) Hybrid reasoning with rules and constraints under wellfounded semantics, In: First international conference on web reasoning and rule systems (RR2007). vol 4524 of lecture notes in computer science, Springer, Innsbruck. Austria, pp 348-357

8. Eiter T, Fink M, Tompits H, Wolttan S (2005) Strong and uniform equivalence in answer-set programming: characterizations and complexity results for the non-gronnd case. In: Proceedings of the twentieth national conference on artificial intelligence and the seventeenth innovative applications of artificial intelligence conference. pp 695-700

9. Eiter T, Ianni G, Schindlauer R. Tonpits $\mathrm{H}$ (2005) A uniform integration of higher-order reasoning and external evaluations in answet-set programming. In: IJCAI 2005. pp 90-96

10. Eiter $T$, Lukasiewicz $T$, Schindlauer $R$, Tompits $H$ (2004) Combining answer set programming with description logics for the semantic web. In: Proceedings of the ninth international conference on principles of knowledge representation and reasoning (KR'04)

11. Ensan F. Du W (2009) A knowledge encapsulation approach to ontology modularization. Knowledge and Information Systems Online First, to appear

12. Ferraris P. Lee J, Lifschitz V (2007) A new perspective on stable models. In: Proceedings of the twentieth international joint conference on artificial intelligence (IJCAI-07), AAAI, Hyderabat, India, pp 372-379

13. Ghilardi S. Lutz C, Wolter F (2006) Did I damage my ontology: a case for conservative extensions of description logics. In: Proceedings of the tenth international conference on principles of knowledge tepresentation and reasoning (KR'06), pp 187-197

14. Heymans $S$ (2006) Decidable open answer set programming. PhD thesis, Theoretical Computer Science Lab (TINF). Department of Computer Science, Vtije Universiteit Brussel, Brussels, Belgium

15. Heymans S, Nieuwenborgh DV. Vermeir D (2005) Guarded open answer set progranming. In: 8th International conference on logic progranming and non monotonic reasoning (LPNMR 2005). volume 3662 in 'LNAI', Springer, pp 92-104

16. Heymans S, Predoiu L, Fejer C, de Bruijn J, van Nieuwenborgh D (2006) G-bybtid knowledge bases. In: Workshop on applications of logic programming in the semantic web and semantic web services (ALPSWS 2006)

17. Jing Y, Jeong D, Baik D-K (2009) SPARQL graph pattern rewriting for OWL-DL inference queries. Knowl Inf Syst 20:243-262

18. Knort M. Alferes J. Hitzler $P$ ( 2008 ) A cohetent well-founded model for hybrid mknf knowledge bases. In: 18th European conference on artificial intelligence (ECAI2008). Volume 178 in 'Frontiers in artificial intelligence and applications*, IOS Press, pp 99-103

19. Lifschitz V. Pearce D, Valverde A (2001) Strongly equivalent logic programs. ACM Trans Comput Log $2(4): 526-541$

20. Lifschitz V, Pearce D, Valverde A (2007) A characterization of strong equivalence for logic programs with variables. In: 9th International conference on logic programming and nonmonotonic reasoning (LPNMR), Vol. 4483 of lecture notes in computer science, Springer, Tempe. AZ. USA. pp 188-200

21. Lifschitz V. Woo T (1992) Answer sets in general nonmonotonic reasoning (preliminary report). In: Nebel B. Rich C, Swartout W (eds) 'KR' 92 . Ptinciples of knowledge representation and reasoning: proceedings of the thitd international conference, Morgan Kanfmann, San Mateo, California, pp 603-614

22. Lin F (2002) Reducing strong equivalence of logic programs to entailment in classical propositional logic. In: Proceedings of the eights international conference on ptinciples of knowledge representation and reasoning (KR'02). pp 170-176

23. Motik B, Rosati R (2007) A faithful integration of description logics with logic progranming. In: Proceedings of the twentieth international joint conference on artificial intelligence (IJCAI-07), AAAI, Hyderabad, India, pp $477-482$

24. Pearce D (1997) A new logical characterization of stable models and answer sets. In: Proceedings of NMELP 96. vol 1216 of lecture notes in computer science. Springer. pp 57-70

25. Pearce D (2006) 'Equilibrium logic'. Ann Math Artif Intell 47:3-41

26. Pearce D, Valverde A (2005) A first-order nonmonotonic extension of constructive logic. Studia Logica $80: 321-346$

27. Pearce D. Valverde A, (2006) Quantfied equilibrium logic. Technical report. Universidad Rey Juan Car los. (in press)

28. Polleres A (2007) From SPARQL to rules (and back), in 'WWW 2007', pp 787-796

29. Rosati $\mathrm{R}(2005)$ On the decidability and complexity of integrating ontologies and rules. J Web Semant $3(1) \div 61-73$

30. Rosati R (2005b) Senantic and computational advantages of the safe integration of ontologies and rules. In: Proceedings of the third international workshop on principles and practice of semantic web reasoning (PPSWR 2005), vol 3703 of lecture notes in computer science. Springer, pp 50-64 
31. Rosati $\mathrm{R}$ (2006) DC + log: tight integration of description logics and disjunctive datalog. In: Proceedings of the tenth international conference on ptinciples of knowledge representation and reasoning (KR'06). pp 68-78

32. van Dalen D (1983) Logic and structure. Springer. Betlin

33. Wang K, Billington D, Blee J, Antoniou G (2004) Combining description logic and defeasible logic for the semantic web. In: Proceedings of the third international workshop rules and rule markup languages for the semantic web (RuleML 2004). vol 3323 of lecture notes in computer science. Springer. pp 170-181

\section{Author Biographies}
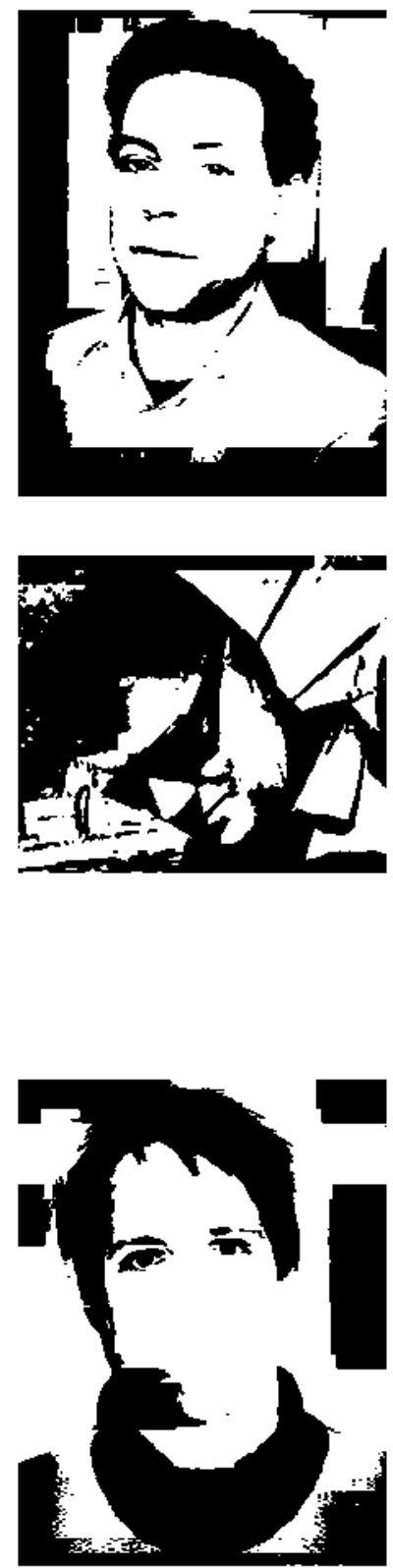

Jos de Bruijn obtained his $\mathrm{PhD}$ of Computer Science from the University of Innsbruck in 2008 . Before joining the Technical University of Vienna in 2010 he worked as Assistant-Professor at the Faculty of Computer Science, Free Utriversity of Bozen-Bolzano. His tesearch interests include combination of logic and logic programming, the telationship between knowledge representation (KR) and database technology, and the application of KR to the Semantic Web.

David Pearce A strong focus of David Pearce' scientific work has been on logic and its application to problems of knowledge representation and reasoning. Until the late $1980 \mathrm{~s}$ this was mainly in the context of classical logic and its extensions, and its application to KRR in sciences such as physics and economics. Thereafter he became interested in problems of common sense teasoning and the application of nonclassical logics to KRR within artificial intelligence. In the mid-1990s be developed a system of nommonotonic reasoning called equilibitum logic. closely related to the stable model semantics of logic prograns that now forms the basis of answer set progranming (ASP). David Pearce curtently works as I3 Professor at the Universidad Politécnica de Madrid, Spain and moreover is a member of the Centro para las Tecnologías Inteligentes de la Información y sus Aplicaciones (CETINIA).

Axel Polleres heads the Semantic Search research stream at the Digital Enterprise Research Institute of NUI Galway, Ireland. His research focus lies in Semantic. Web Search, Rules Languages, Query answering and Reasoning ovet Semantic Web Data. He obtained his PhD at TU Vienna in 2003 and worked as postdoctoral researcer at the Unjversity of Innsbiuck, Austria and as research fellow at Unjersidad Rey Juan Carlos. Spain since. Moreover, Axel is active in the W3C standardisation activities as former member of the W3C advisory committee, member and standards' co-editor of the W3C RIF working group and co-chair of the W3C SPARQL1.l working group. 


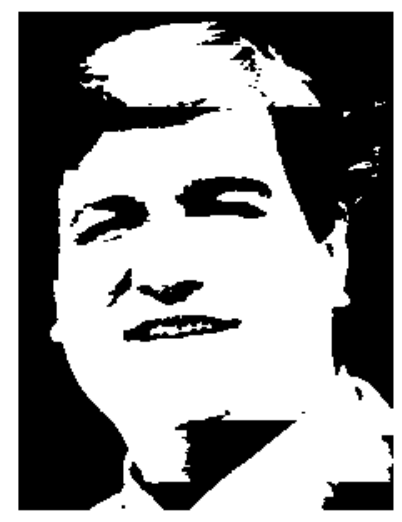

Agustill Valverde has been a member of the Dpt. of Applied Mathematics at the University of Málaga (Spain) since 1991. He received his $\mathrm{PhD}$ in Mathentics in 1996 . with a thesis about Automated Reasoning in Finite Valued Logics, and he became Associate Professot in 2001. His research is devored to the use of formal methods in Computer Science and Computational Logic. with special emphasis on automated deduction methods for classical and non-classical logics. Recent works have focused on equilibrium logic. a system of nonmonotonic reasoning that forms a logical basis for Answer Set Programming. 